\title{
OPTIMAL LOW-THRUST TAKEOFF FROM AN ORBIT ABOUT
}

\author{
AN OBLATE PLANET*
}

\author{
ROBERT A. JACOBSON \\ Senior Engineer, Mission Analysis Division, Jet Propulsion Laboratory, \\ Pasadena, Calif. 91103, U.S.A.
}

and

WILLIAM F. POWERS

Associate Professor, Department of Aerospace Engineering, The University of Michigan, Ann Arbor, Mich. 48104, U.S.A.

(Received 19 August, 1975)

\begin{abstract}
Future space missions to the outer planets may depend upon the use of low-thrust propulsion systems. As these planets are decidedly oblate, the question of the effect of that oblateness on a low-thrust trajectory is of some interest. In this paper the problem of optimal energy increase is attacked under the assumption that the coefficients for the second zonal harmonic, i.e., $J_{2}$, and the nondimensional thrust acceleration are the same order of magnitude. Using a two variable asymptotic expansion technique, a near optimal control program is generated and the first order uniformly valid approximation for the corresponding trajectory is obtained. Tangential thrust is shown to be a good near-optimal thrust program even in the presence of oblateness effects. The optimal control program is found to be oscillatory and quite similar to the optimal control for energy increase in an inverse square gravitational field.
\end{abstract}

\section{Introduction}

The problem of takeoff from planetary orbit by a low thrust spacecraft has been the subject of numerous numerical and analytical studies. For the case of an inverse square gravity field, solutions have been generated for specified thrust programs, i.e., circumferential thrust (Tsien, 1953), tangential thrust (Benny, 1958), arbitrary constant angle thrust (Ting and Brofman, 1954), radial thrust (Dobrowolski, 1958 and Tsien, 1953), and for optimal thrust programs (Breakwell and Rausch, 1966; Jacobson and Powers, 1971, 1972; Lawden, 1958). For an oblate gravity field in which the nondimensional thrust acceleration is of the same order as the oblateness coefficient for the second zonal harmonic, Zee has obtained solutions for the cases of tangential (Zee, 1968) and radial thrust (Zee, 1969).

The problem considered here is that of optimal takeoff from an orbit about an oblate planet. The spacecraft is assumed to operate with a constant thrust acceleration of the same order as that assumed by Zee. Since the thrust acceleration is constant, the only control is thrust direction, and the fuel optimal and time optimal trajectories

* This research was supported by the National Aeronautics and Space Administration under Grant NGR-23-005-329. 
coincide. The particular optimal takeoff trajectory to be studied is the one on which a specified terminal energy is acquired in minimum time (or equivalently, with minimum fuel expenditure). One example of such a trajectory is the optimal escape trajectory. However, the method of solution requires the gravitational force to remain dominant; and consequently, the results of the analysis are valid only for trajectories attaining final energies somewhat less than escape energy. This restriction on final energy is encountered on all analytical solutions to low thrust takeoff problems.

\section{Mathematical Formulation}

\section{A. COORDINATE SYSTEM AND EQUATIONS OF MOTION}

The motion of the vehicle is described relative to a coordinate system which is rotating with an angular velocity and acceleration that are determined as part of the problem solution. Figure 1 shows the relation between this system and a planet centered inertial system. The angular velocity and acceleration vectors are aligned with the inertial $Z$ axis, and the rotation is performed with the $x y$ plane inclined at a constant angle to the inertial $X Y$ plane. The angle of inclination is determined at the initial time by requiring the $x y$ plane to coincide with the osculating orbital plane. It should be emphasized, however, that after the initial time the $x y$ plane and osculating orbital plane are not coincident.

Within the rotating system the motion is expressed in cylindrical coordinates, and

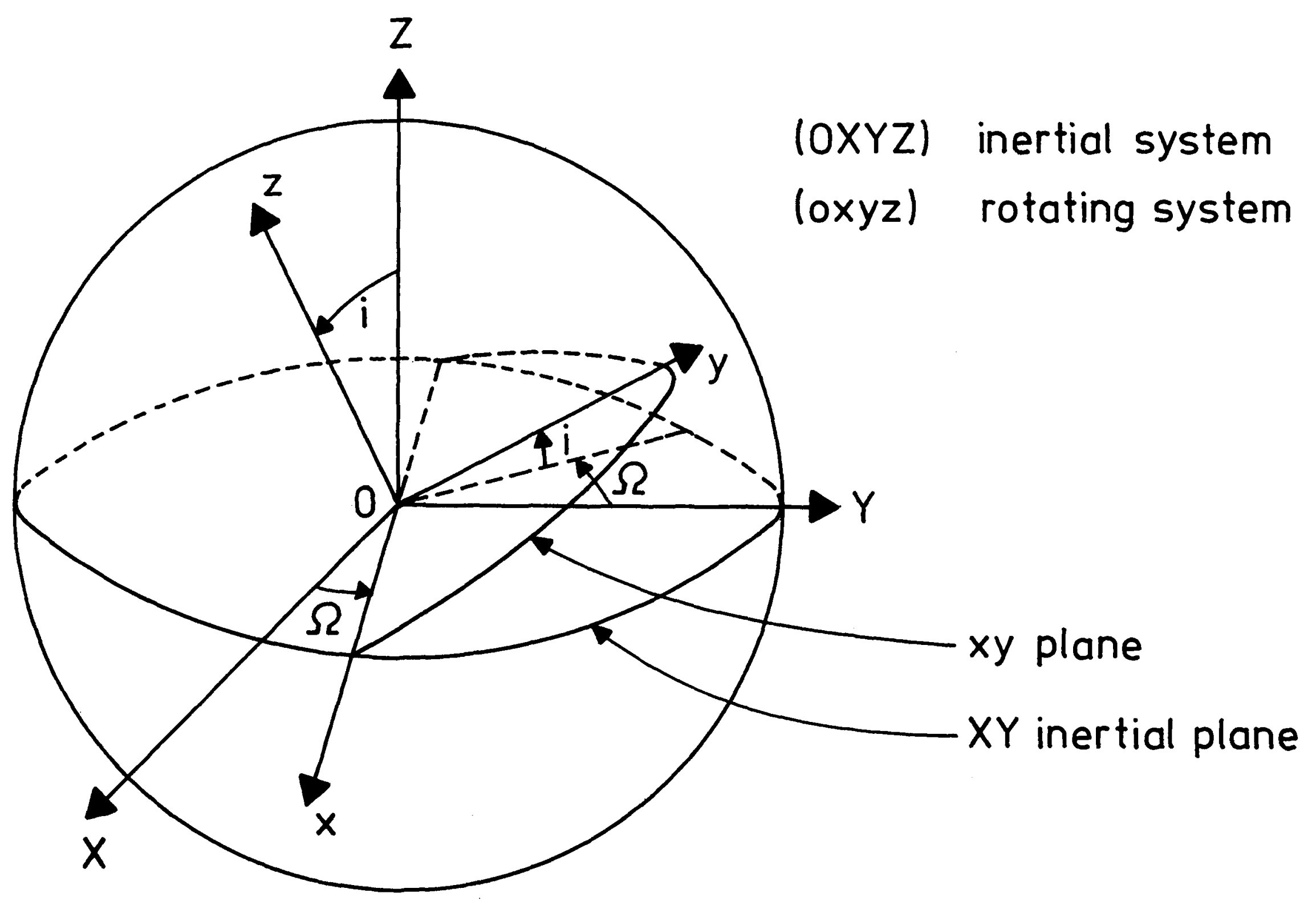

Fig. 1. 
the thrust acceleration vector is defined with respect to the unit vectors for those coordinates (Figure 2).

$$
\mathbf{a}=a\left[(\cos \psi \sin \alpha) \hat{e}_{r}+(\cos \psi \cos \alpha) \hat{e}_{\theta}+(\sin \psi) \hat{e}_{z}\right] .
$$

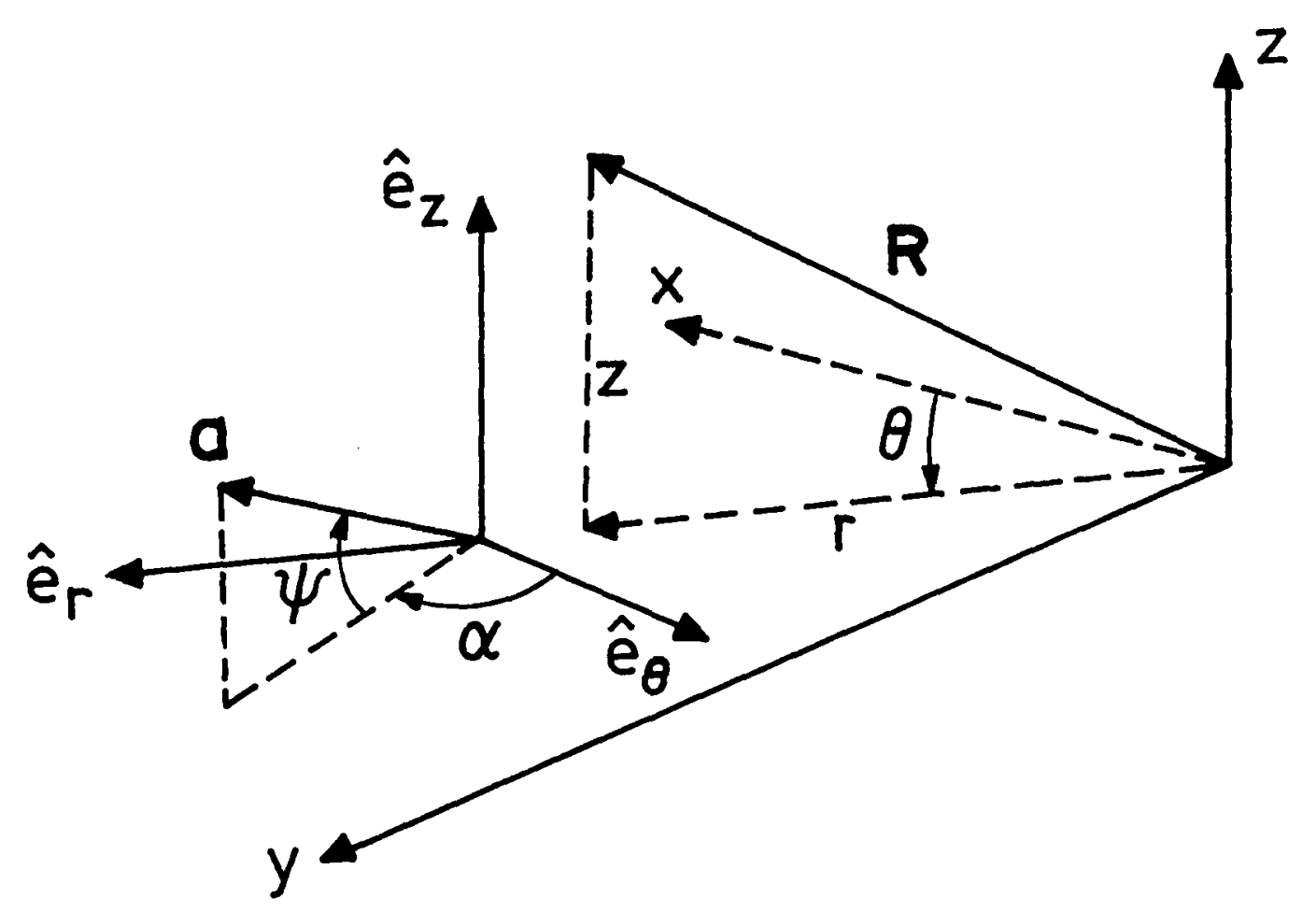

Fig. 2.

Non-dimensionalizing the equations of motion with respect to an unperturbed circular orbit of radius $R_{0}$ leads to the new independent variable

$$
\tau=t \sqrt{\frac{\mu}{R_{0}^{3}}}
$$

and introduces the parameters

$$
\hat{a}=\frac{a}{\left(\mu / R_{0}^{2}\right)}, \quad \varepsilon=J_{2}\left(\frac{R_{\otimes}}{R_{0}}\right)^{2},
$$

where

$\mu=$ gravitational constant of the planet,

$R_{\otimes}=$ planetary equatorial radius,

$J_{2}=$ oblateness coefficient,

$a=$ thrust acceleration magnitude.

The parameter $\hat{a}$ is the nondimensional thrust acceleration and the parameter $\varepsilon$ scales the acceleration due to planetary oblateness. For the remainder of the paper it will be assumed that $\hat{a}$ and $\varepsilon$ are of the same order of magnitude, and the relation

$$
\hat{a}=\kappa \varepsilon,
$$

where $\kappa=0(1)$ will be used to eliminate $\hat{a}$ from the equations of motion. Note that alternatively the relation $\varepsilon=\hat{\kappa} \hat{a}$ could be used, and $\varepsilon$ could be eliminated in lieu of $\hat{a}$. However (1.2) has been selected because, by setting $\kappa$ to zero, it permits the equations of motion to be reduced to those of a non-thrusting vehicle in an oblate gravity field.

The complete non-dimensional set of equations of motion is then 


$$
\begin{aligned}
& \frac{\mathrm{d} r}{\mathrm{~d} \tau}=V \sin \gamma \\
& \frac{\mathrm{d} \theta}{\mathrm{d} \tau}=\frac{V}{r} \cos \gamma \\
& \frac{\mathrm{d} z}{\mathrm{~d} \tau}=w \\
& \frac{\mathrm{d} V}{\mathrm{~d} \tau}=-\frac{r \sin \gamma}{\left(r^{2}+z^{2}\right)^{3 / 2}}+\kappa \varepsilon \cos \psi \cos \varphi-\frac{3}{2} \frac{\varepsilon}{\left(r^{2}+z^{2}\right)^{5 / 2}} \times \\
& \times\left[r\left(1+\sin ^{2} i\right) \sin \gamma+r \sin ^{2} i \sin (2 \theta-\gamma)+\right. \\
& +z \sin 2 i \cos (\theta-\gamma)]+\frac{15}{2} \frac{\varepsilon r \sin \gamma}{\left(r^{2}+z^{2}\right)^{7 / 2}}(r \sin i \sin \theta+z \cos i)^{2}+ \\
& +2 \frac{\mathrm{d} \Omega}{\mathrm{d} \tau} w \sin i \sin (\theta-\gamma)-\frac{1}{2}\left(\frac{\mathrm{d} \Omega}{\mathrm{d} \tau}\right)^{2} \times \\
& \times\left[z \sin 2 i \cos (\theta-\gamma)+r \sin ^{2} i \sin (2 \theta-\gamma)+\right. \\
& \left.+r\left(1+\cos ^{2} i\right) \sin \gamma\right]-\frac{1}{2} \frac{\mathrm{d}^{2} \Omega}{\mathrm{d} \tau^{2}} \times \\
& \times[r \cos i \cos \gamma-z \sin i \sin (\theta-\gamma)], \\
& \frac{\mathrm{d} \gamma}{\mathrm{d} \tau}=\frac{V}{r} \cos \gamma-\frac{r \cos \gamma}{V\left(r^{2}+z^{2}\right)^{3 / 2}}+\frac{\kappa \varepsilon}{V} \cos \psi \sin \phi- \\
& -\frac{3}{2} \frac{\varepsilon}{V\left(r^{2}+z^{2}\right)^{5 / 2}}\left[r\left(1+\sin ^{2} i\right) \cos \gamma-r \sin ^{2} i \times\right. \\
& \times \cos (2 \theta-\gamma)+z \sin 2 i \sin (\theta-\gamma)]+ \\
& +\frac{15}{2} \frac{\varepsilon r \cos \gamma}{V\left(r^{2}+z^{2}\right)^{7 / 2}}(r \sin i \sin \theta+z \cos i)^{2}- \\
& -\frac{2}{V} \frac{\mathrm{d} \Omega}{\mathrm{d} \tau}[w \sin i \cos (\theta-\gamma)-V \cos i]- \\
& -\frac{1}{2 V}\left(\frac{\mathrm{d} \Omega}{\mathrm{d} \tau}\right)^{2}\left[z \sin 2 i \sin (\theta-\gamma)-r\left(1+\cos ^{2} i\right) \cos \gamma-\right. \\
& \left.-r \sin ^{2} i \cos (2 \theta-\gamma)\right]+\frac{1}{V} \frac{\mathrm{d}^{2} \Omega}{\mathrm{d} \tau^{2}} \times \\
& \times[r \cos i \sin \gamma-z \sin i \cos (\theta-\gamma)],
\end{aligned}
$$




$$
\begin{aligned}
\frac{\mathrm{d} w}{\mathrm{~d} \tau}= & \frac{-z}{\left(r^{2}+z^{2}\right)^{3 / 2}}+\kappa \varepsilon \sin \psi-\frac{3}{2} \frac{\varepsilon}{\left(r^{2}+z^{2}\right)^{5 / 2}}\left[z\left(1+2 \cos ^{2} i\right)+\right. \\
& +r \sin 2 i \sin \theta]+\frac{15}{2} \frac{\varepsilon z}{\left(r^{2}+z^{2}\right)^{7 / 2}}(r \sin i \sin \theta+ \\
& +z \cos i)^{2}-2 \frac{\mathrm{d} \Omega}{\mathrm{d} \tau} V \sin i \sin (\theta-\gamma)- \\
& -\frac{1}{2}\left(\frac{\mathrm{d} \Omega}{\mathrm{d} \tau}\right)^{2}\left(r \sin 2 i \sin \theta-2 z \sin ^{2} i\right)+\frac{\mathrm{d}^{2} \Omega}{\mathrm{d} \tau^{2}}(r \sin i \cos \theta)
\end{aligned}
$$

where

$r=$ component of position vector in $x y$ plane,

$\theta=$ angle from $x$ axis to $r$,

$z=$ component of position vector normal to $x y$ plane,

$V=$ magnitude of component of velocity vector in $x y$ plane,

$\gamma=$ angle between unit vector $\hat{e}_{\theta}$ and component of velocity vector in $x y$ plane,

$w=$ component of velocity vector normal to $x y$ plane,

$i=$ angle of inclination of $x y$ plane to $X Y$ plane,

$\Omega=$ angle of rotation of (oxyz) system measured from $X$ axis to $x$ axis,

$\phi=\alpha-\gamma=$ angle defining direction of component of thrust acceleration vector in $x y$ plane,

$\psi=$ angle defining component of thrust acceleration vector normal to $x y$ plane.

\section{B. OPTIMIZATION PROBLEM FORMULATION}

The optimal control programs $\phi(\tau)$ and $\psi(\tau)$ are found by application of the calculus of variations, with the performance index and Hamiltonian

$$
\begin{aligned}
& J=\tau_{f}-\tau_{0}, \\
& H=\lambda_{1} \frac{\mathrm{d} r}{\mathrm{~d} \tau}+\lambda_{2} \frac{\mathrm{d} \theta}{\mathrm{d} \tau}+\lambda_{3} \frac{\mathrm{d} z}{\mathrm{~d} \tau}+\lambda_{4} \frac{\mathrm{d} V}{\mathrm{~d} \tau}+\lambda_{5} \frac{\mathrm{d} \gamma}{\mathrm{d} \tau}+\lambda_{6} \frac{\mathrm{d} w}{\mathrm{~d} \tau} .
\end{aligned}
$$

The resulting optimal controls are

$$
\begin{aligned}
& \sin \phi=\frac{\lambda_{5}}{\sqrt{\lambda_{5}^{2}+\lambda_{4}^{2} V^{2}}}, \quad \cos \phi=\frac{\lambda_{4} V}{\sqrt{\lambda_{5}^{2}+\lambda_{4}^{2} V^{2}}} \\
& \sin \psi=\frac{\lambda_{6} V}{\sqrt{\lambda_{5}^{2}+\lambda_{4}^{2} V^{2}+\lambda_{6}^{2} V^{2}}}, \quad \cos \psi=\frac{\sqrt{\lambda_{5}^{2}+\lambda_{4}^{2} V^{2}}}{\sqrt{\lambda_{5}^{2}+\lambda_{4}^{2} V^{2}+\lambda_{6}^{2} V^{2}}}
\end{aligned}
$$


and

$$
-\pi<\phi<\pi, \quad-\frac{\pi}{2}<\psi<\frac{\pi}{2} .
$$

The necessary conditions require the multipliers to satisfy the equations

$$
\begin{array}{ll}
\frac{\mathrm{d} \lambda_{1}}{\mathrm{~d} \tau}=-\frac{\partial H}{\partial r}, & \frac{\mathrm{d} \lambda_{4}}{\mathrm{~d} \tau}=-\frac{\partial H}{\partial V} \\
\frac{\mathrm{d} \lambda_{2}}{\mathrm{~d} \tau}=-\frac{\partial H}{\partial \theta}, & \frac{\mathrm{d} \lambda_{5}}{\mathrm{~d} \tau}=-\frac{\partial H}{\partial \gamma} \\
\frac{\mathrm{d} \lambda_{3}}{\mathrm{~d} \tau}=-\frac{\partial H}{\partial z}, & \frac{\mathrm{d} \lambda_{6}}{\mathrm{~d} \tau}=-\frac{\partial H}{\partial w} .
\end{array}
$$

Due to the complexity of these equations, they will not be given explicitly in this paper. Their development, which follows from (1.10) and (1.3)-(1.8), however, is straight-forward, though tedious.

The specified boundary conditions on the problem are

$$
\begin{aligned}
& \tau_{0}=0 ; \\
& r(0)=1, \quad V(0)=1-\frac{\mathrm{d} \Omega}{\mathrm{d} \tau} \cos i, \\
& \theta(0)=0, \quad \gamma(0)=0, \\
& z(0)=0, \quad w(0)=\frac{\mathrm{d} \Omega}{\mathrm{d} \tau} \sin i \\
& E\left(\tau_{f}\right)=\frac{1}{2}\left[\left(V \sin \gamma+z \frac{\mathrm{d} \Omega}{\mathrm{d} \tau} \sin i \cos \theta\right)^{2}+\right. \\
& +\left(V \cos \gamma+r \frac{\mathrm{d} \Omega}{\mathrm{d} \tau} \cos i-z \frac{\mathrm{d} \Omega}{\mathrm{d} \tau} \sin i \sin \theta\right)^{2}+ \\
& \left.+\left(w-r \frac{\mathrm{d} \Omega}{\mathrm{d} \tau} \sin i \cos \theta\right)^{2}\right]_{\tau_{f}}- \\
& -\frac{1}{R}\left[1+\frac{1}{2} \varepsilon\left(1-3 \sin ^{2} \delta\right)\right]_{\tau_{f}}=E_{f},
\end{aligned}
$$

where

$$
\begin{aligned}
& R=\sqrt{r^{2}+z^{2}} \\
& \sin \delta=\frac{r \sin i \sin \theta+z \cos i}{\sqrt{r^{2}+z^{2}}}
\end{aligned}
$$

$E_{f}=$ specified terminal energy level. 
The initial conditions on $V, \gamma$, and $w$ correspond to an inertial velocity $\dot{\mathbf{R}}(0)=1 \hat{e}_{\theta}$. The initial $\theta$ value places the vehicle in the equatorial plane; this position choice reduces the complexity of the analysis, although the solution procedures can be followed for any choice of initial $\theta$.

Since the twelfth order two-point boundary value problem posed by Equations (1.3)-(1.8) and (1.13), requires fourteen boundary conditions, the remaining six must be obtained from the transversality conditions. These conditions are

$$
\begin{aligned}
& \lambda_{1}\left(\tau_{f}\right)-\lambda_{4}\left(\tau_{f}\right)\left(\frac{\partial E}{\partial V}\right)_{\tau_{f}}^{-1}\left(\frac{\partial E}{\partial r}\right)_{\tau_{f}}=0 \\
& \lambda_{2}\left(\tau_{f}\right)-\lambda_{4}\left(\tau_{f}\right)\left(\frac{\partial E}{\partial V}\right)_{\tau_{f}}^{-1}\left(\frac{\partial E}{\partial \theta}\right)_{\tau_{f}}=0 \\
& \lambda_{3}\left(\tau_{f}\right)-\lambda_{4}\left(\tau_{f}\right)\left(\frac{\partial E}{\partial V}\right)_{\tau_{f}}^{-1}\left(\frac{\partial E}{\partial z}\right)_{\tau_{f}}=0 \\
& \lambda_{5}\left(\tau_{f}\right)-\lambda_{4}\left(\tau_{f}\right)\left(\frac{\partial E}{\partial V}\right)_{\tau_{f}}^{-1}\left(\frac{\partial E}{\partial \gamma}\right)_{\tau_{f}}=0 \\
& \lambda_{6}\left(\tau_{f}\right)-\lambda_{4}\left(\tau_{f}\right)\left(\frac{\partial E}{\partial V}\right)_{\tau_{f}}^{-1}\left(\frac{\partial E}{\partial w}\right)_{\tau_{f}}=0 \\
& \lambda_{4}\left(\tau_{f}\right)\left(\frac{\partial E}{\partial V}\right)_{\tau_{f}}^{-1}\left(\mathbf{a} \cdot \dot{\mathbf{R}}+\frac{\partial E}{\partial \tau}\right)_{\tau_{f}}=1,
\end{aligned}
$$

where

$$
\begin{aligned}
\dot{\mathbf{R}}= & \left(V \sin \gamma+z \frac{\mathrm{d} \Omega}{\mathrm{d} \tau} \sin i \cos \theta\right) \hat{e}_{r}+ \\
& +\left(V \cos \gamma+r \frac{\mathrm{d} \Omega}{\mathrm{d} \tau} \cos i-z \frac{\mathrm{d} \Omega}{\mathrm{d} \tau} \sin i \sin \theta\right) \hat{e}_{\theta}+ \\
& +\left(w-r \frac{\mathrm{d} \Omega}{\mathrm{d} \tau} \sin i \cos \theta\right) \hat{e}_{z} .
\end{aligned}
$$

The partial derivatives of the energy $E$ are given in Appendix B.

The specific problem of determining the optimal controls to take the vehicle to energy level $E_{f}$ in minimum time is now formulated as a two point boundary value problem with seven conditions at each boundary.

\section{Asymptotic Solution}

\section{A. GENERAL METHOD}

Since the solution of the problem posed in I.B cannot be found analytically, it will be approximated with a general asymptotic expansion which is developed by a method of 
multiple scales (Cole, 1968). The problem has two readily identifiable and distinct characteristic time constants

$$
\begin{aligned}
& T_{1}=\sqrt{R_{0}^{3} / \varepsilon \mu}, \\
& T_{2}=\sqrt{R_{0}^{3} / \mu},
\end{aligned}
$$

where $T_{1}$ corresponds to the time scale on which the orbit deviates from the initial circular, and $T_{2}$ corresponds to the time scale on which the vehicle moves along its orbit. As a consequence, two new independent variables for the problem can be defined

$$
\tau_{1}=\varepsilon \tau, \quad \tau_{2}=\int_{0}^{\tau} \omega_{0}(\varepsilon s) \mathrm{d} s
$$

where $\omega_{0}(\cdot)$ is a function which is to be determined in the process of generating the expansions.

The direct approach would now assume the state variables and multipliers to be represented by expansions of the form

$$
q=q\left(\tau_{1}, \tau_{2}\right)=\sum_{n=0}^{N} \varepsilon^{n} q_{n}\left(\tau_{1}, \tau_{2}\right)+O\left(\varepsilon^{N+1}\right)
$$

and these expansions would be substituted into Equations (1.3)-(1.8), (1.13), and the boundary conditions in order to generate equations and boundary conditions for the terms in the expansions. However, to be valid such an expansion must satisfy the equations for the case $\varepsilon=0$, and in our problem the final transversality condition is degenerate at $\varepsilon=0$. As a result, any solution satisfying that condition and found by direct application of (2.4) possesses a singularity. The source of this singularity is obvious for if $\varepsilon=0$, then $\kappa \varepsilon=\hat{a}=0$ and the optimal control problem does not exist, i.e., there is no control available for the vehicle. In order to develop a properly posed problem for solution by an asymptotic method, a simple scaling of the multipliers is employed

$$
\lambda \equiv \frac{1}{\kappa \varepsilon} \lambda
$$

Since the multiplier Equations (1.13), control Equations (1.11)-(1.12), and first five transversality conditions are homogeneous in the multipliers, they remain unchanged. The troublesome final transversality condition, however, becomes

$$
\lambda_{4}\left(\tau_{f}\right)\left(\frac{\partial E}{\partial V}\right)_{\tau_{f}}^{-1}\left(\mathbf{a} \cdot \dot{\mathbf{R}}+\frac{\partial E}{\partial \tau}\right)_{\tau_{f}}=\kappa \varepsilon
$$

Now a straight-forward substitution of expansions of the form (2.4) into the Equations (1.3)-(1.8), (1.11)-(1.12), (1.13), and boundary conditions (1.14), (1.15), 
(2.6) may be used to form a system of equations for the expansion terms. Since we are assuming the expansions to be functions of $\tau_{1}$ and $\tau_{2}$, the derivatives with respect to $\tau$ must be replaced by

$$
\frac{\mathrm{d}(\cdot)}{\mathrm{d} \tau}=\frac{\partial(\cdot)}{\partial \tau_{1}} \frac{\mathrm{d} \tau_{1}}{\mathrm{~d} \tau}+\frac{\partial(\cdot)}{\partial \tau_{2}} \frac{\mathrm{d} \tau_{2}}{\mathrm{~d} \tau}=\varepsilon \frac{\partial(\cdot)}{\partial \tau_{1}}+\omega_{0}\left(\tau_{1}\right) \frac{\partial(\cdot)}{\partial \tau_{2}} .
$$

In addition, in order to reduce the complexity of the equations, the expanded functions of the control angles will be represented by

$$
\begin{aligned}
& \cos \phi=\alpha_{0}\left(\tau_{1}, \tau_{2}\right)+\varepsilon \alpha_{1}\left(\tau_{1}, \tau_{2}\right)+\varepsilon^{2} \alpha_{2}\left(\tau_{1}, \tau_{2}\right)+\cdots \\
& \sin \phi=\beta_{0}\left(\tau_{1}, \tau_{2}\right)+\varepsilon \beta_{1}\left(\tau_{1}, \tau_{2}\right)+\varepsilon^{2} \beta_{2}\left(\tau_{1}, \tau_{2}\right)+\cdots \\
& \cos \psi=\delta_{0}\left(\tau_{1}, \tau_{2}\right)+\varepsilon \delta_{1}\left(\tau_{1}, \tau_{2}\right)+\varepsilon^{2} \delta_{2}\left(\tau_{1}, \tau_{2}\right)+\cdots \\
& \sin \psi=v_{0}\left(\tau_{1}, \tau_{2}\right)+\varepsilon v_{1}\left(\tau_{1}, \tau_{2}\right)+\varepsilon^{2} v_{2}\left(\tau_{1}, \tau_{2}\right)+\cdots,
\end{aligned}
$$

where $\alpha_{i}, \beta_{i}, \delta_{i}, v_{i}$ are given in terms of the multipliers in Appendix A.

The equations of motion also contain one unknown function $\Omega(\tau)$, the rotation angle of the coordinate system. It will be assumed to be a function of the slow time variable $\tau_{1}$ only, and to possess the expansion

$$
\Omega(\tau)=\Omega\left(\tau_{1}\right)=\Omega_{0}\left(\tau_{1}\right)+\varepsilon \Omega_{1}\left(\tau_{1}\right)+\varepsilon^{2} \Omega_{2}\left(\tau_{1}\right)+\cdots .
$$

B. ZERO ORDER STATE AND MULTIPLIER SOLUTION

The zero order state and multiplier equations are

$$
\begin{aligned}
\omega_{0} \frac{\partial r_{0}}{\partial \tau_{2}}= & V_{0} \sin \gamma_{0}, \\
\omega_{0} \frac{\partial \theta_{0}}{\partial \tau_{2}}= & \frac{V_{0}}{r_{0}} \cos \gamma_{0} \\
\omega_{0} \frac{\partial z_{0}}{\partial \tau_{2}}= & w_{0}, \\
\omega_{0} \frac{\partial V_{0}}{\partial \tau_{2}}= & -\frac{r_{0} \sin \gamma_{0}}{\left(r_{0}^{2}+z_{0}^{2}\right)^{3 / 2}}, \\
\omega_{0} \frac{\partial \gamma_{0}}{\partial \tau_{2}}= & \frac{1}{V_{0}}\left[\frac{V_{0}^{2}}{r_{0}}-\frac{r_{0}}{\left(r_{0}^{2}+z_{0}^{2}\right)^{3 / 2}}\right] \cos \gamma_{0}, \\
\omega_{0} \frac{\partial w_{0}}{\partial \tau_{2}}= & -\frac{z_{0}}{\left(r_{0}^{2}+z_{0}^{2}\right)^{3 / 2}}, \\
\omega_{0} \frac{\partial \lambda_{10}}{\partial \tau_{2}}= & \left(\frac{V_{0}}{r_{0}^{2}} \cos \gamma_{0}\right) \lambda_{20}-\left[\frac{2 r_{0}^{2}-z_{0}^{2}}{\left(r_{0}^{2}+z_{0}^{2}\right)^{5 / 2}} \sin \gamma_{0}\right] \lambda_{40}- \\
& -\left[\frac{2 r_{0}^{2}-z_{0}^{2}}{\left(r_{0}^{2}+z_{0}^{2}\right)^{5 / 2}}-\frac{V_{0}}{r_{0}^{2}}\right] \cos \gamma_{0} \lambda_{50}-\left[\frac{3 r_{0} z_{0}}{\left(r_{0}^{2}+z_{0}^{2}\right)^{5 / 2}}\right] \lambda_{60},
\end{aligned}
$$




$$
\begin{aligned}
\omega_{0} \frac{\partial \lambda_{20}}{\partial \tau_{2}}= & 0 \\
\omega_{0} \frac{\partial \lambda_{30}}{\partial \tau_{2}}= & -\left[\frac{3 r_{0} z_{0} \sin \gamma_{0}}{\left(r_{0}^{2}+z_{0}^{2}\right)^{5 / 2}}\right] \lambda_{40}-\left[\frac{3 r_{0} z_{0} \cos \gamma_{0}}{V_{0}\left(r_{0}^{2}+z_{0}^{2}\right)^{5 / 2}}\right] \lambda_{50}- \\
& -\left[\frac{2 z_{0}^{2}-r_{0}^{2}}{\left(r_{0}^{2}+z_{0}^{2}\right)^{5 / 2}}\right] \lambda_{60} \\
& -\left[\frac{3 r_{0} z_{0} \cos \gamma_{0}}{V_{0}\left(r_{0}^{2}+z_{0}^{2}\right)^{5 / 2}}\right] \lambda_{50}, \\
\omega_{0} \frac{\partial \lambda_{40}}{\partial \tau_{2}}= & -\left(\sin \gamma_{0}\right) \lambda_{10}-\left(\frac{1}{r_{0}} \cos \gamma_{0}\right) \lambda_{20}-\left[\frac{3 r_{0} z_{0} \sin \gamma_{0}}{\left(r_{0}^{2}+z_{0}^{2}\right)^{5 / 2}}\right] \lambda_{40}- \\
\omega_{0} \frac{\partial \lambda_{50}}{\partial \tau_{2}}= & -\left(V_{0} \cos \gamma_{0}\right) \lambda_{10}+\left(\frac{V_{0}}{r_{0}} \sin \gamma_{0}\right) \lambda_{20}+\left[\frac{r_{0} \cos \gamma_{0}}{\left(r_{0}^{2}+z_{0}^{2}\right)^{3 / 2}}\right] \lambda_{40}- \\
& -\left[\frac{r_{0} \sin \gamma_{0}}{V_{0}\left(r_{0}^{2}+z_{0}^{2}\right)^{3 / 2}}-\frac{V_{0}}{r_{0}} \sin \gamma_{0}\right] \lambda_{50}, \\
\omega_{0} \frac{\partial \lambda_{60}}{\partial \tau_{2}}= & -\lambda_{30},
\end{aligned}
$$

and the corresponding boundary conditions are

$$
\begin{aligned}
& r_{0}(0,0)=1, \quad V_{0}(0,0)=1, \\
& \theta_{0}(0,0)=0, \quad \gamma_{0}(0,0)=0, \\
& z_{0}(0,0)=0, \quad w_{0}(0,0)=0 \\
& \lambda_{10}\left(\tau_{1 f}, \tau_{2 f}\right)-\lambda_{40}\left(\tau_{1 f}, \tau_{2 f}\right) \omega_{0}\left(\tau_{1 f}\right)=0 \\
& \lambda_{20}\left(\tau_{1 f}, \tau_{2 f}\right)=0, \\
& \lambda_{30}\left(\tau_{1 f}, \tau_{2 f}\right)=0, \\
& \lambda_{40}\left(\tau_{1 f}, \tau_{2 f}\right)=1, \\
& \lambda_{50}\left(\tau_{1 f}, \tau_{2 f}\right)=0, \\
& \lambda_{60}\left(\tau_{1 f}, \tau_{2 f}\right)=0 .
\end{aligned}
$$

One possible solution to the zero order state equations is

$$
\begin{aligned}
& r_{0}\left(\tau_{1}, \tau_{2}\right)=\omega_{0}^{-2 / 3}\left(\tau_{1}\right), \\
& \theta_{0}\left(\tau_{1}, \tau_{2}\right)=\tau_{2}+\Theta_{0}\left(\tau_{1}\right), \\
& z_{0}\left(\tau_{1}, \tau_{2}\right)=0 \\
& V_{0}\left(\tau_{1}, \tau_{2}\right)=\omega_{0}^{1 / 3}\left(\tau_{1}\right), \\
& \gamma_{0}\left(\tau_{1}, \tau_{2}\right)=0 \\
& w_{0}\left(\tau_{1}, \tau_{2}\right)=0
\end{aligned}
$$


The corresponding multiplier solution is

$$
\begin{aligned}
\lambda_{10}\left(\tau_{1}, \tau_{2}\right)= & C_{11}\left(\tau_{1}\right) \omega_{0}\left(\tau_{1}\right)+3 \omega_{0}^{2 / 3}\left(\tau_{1}\right) C_{21}\left(\tau_{1}\right) \tau_{2}- \\
& -\omega_{0}^{2 / 3}\left(\tau_{1}\right)\left[A_{51}\left(\tau_{1}\right) \sin \tau_{2}-B_{51}\left(\tau_{1}\right) \cos \tau_{2}\right], \\
\lambda_{20}\left(\tau_{1}, \tau_{2}\right)= & C_{21}\left(\tau_{1}\right), \\
\lambda_{30}\left(\tau_{1}, \tau_{2}\right)= & A_{31}\left(\tau_{1}\right) \cos \tau_{2}+B_{31}\left(\tau_{1}\right) \sin \tau_{2}, \\
\lambda_{40}\left(\tau_{1}, \tau_{2}\right)= & C_{11}\left(\tau_{1}\right)+3 \omega_{0}^{-1 / 3}\left(\tau_{1}\right) C_{21}\left(\tau_{1}\right) \tau_{2}- \\
& -2 \omega_{0}^{-1 / 3}\left(\tau_{1}\right)\left[A_{51}\left(\tau_{1}\right) \sin \tau_{2}-B_{51}\left(\tau_{1}\right) \cos \tau_{2}\right], \\
\lambda_{50}\left(\tau_{1}, \tau_{2}\right)= & -2 C_{21}\left(\tau_{1}\right)+A_{51}\left(\tau_{1}\right) \cos \tau_{2}+B_{51}\left(\tau_{1}\right) \sin \tau_{2}, \\
\lambda_{60}\left(\tau_{1}, \tau_{2}\right)= & -\omega_{0}^{-1}\left(\tau_{1}\right)\left[A_{31}\left(\tau_{1}\right) \sin \tau_{2}-B_{31}\left(\tau_{1}\right) \cos \tau_{2}\right] .
\end{aligned}
$$

The boundary conditions impose the following conditions on the functions of the slow variable

$$
\begin{aligned}
& \omega_{0}(0)=1, \\
& \Theta_{0}(0)=0, \\
& A_{31}\left(\tau_{1 f}\right)=B_{31}\left(\tau_{1 f}\right)=0, \\
& A_{51}\left(\tau_{1 f}\right)=B_{51}\left(\tau_{1 f}\right)=0, \\
& C_{21}\left(\tau_{1 f}\right)=0, \\
& C_{11}\left(\tau_{1 f}\right)=1 .
\end{aligned}
$$

In order to have a uniformly valid solution, the functions of $\tau_{1}$ must be chosen in a manner which removes unbounded $\tau_{2}$ terms from the expansions and yet permits satisfaction of the boundary conditions. It follows immediately, that

$$
C_{21}\left(\tau_{1}\right) \equiv 0 .
$$

The first order equations must be used to determine the remaining functions of $\tau_{1}$.

\section{FIRST ORDER STATE AND MULTIPLIER SOLUTION}

After the substitution of the zero order state solution into the general expanded first order state equations, they reduce to

$$
\begin{aligned}
& \omega_{0} \frac{\partial r_{1}}{\partial \tau_{2}}=-\frac{\partial r_{0}}{\partial \tau_{1}}+\omega_{0}^{1 / 3} \gamma_{1}, \\
& \omega_{0} \frac{\partial \theta_{1}}{\partial \tau_{2}}=-\frac{\partial \theta_{0}}{\partial \tau_{1}}+\omega_{0}^{2 / 3} V_{1}-\omega_{0}^{5 / 3} r_{1}, \\
& \omega_{0} \frac{\partial z_{1}}{\partial \tau_{2}}=-\frac{\partial z_{0}}{\partial \tau_{1}}+w_{1},
\end{aligned}
$$




$$
\begin{aligned}
\omega_{0} \frac{\partial V_{1}}{\partial \tau_{2}}= & -\frac{\partial V_{0}}{\partial \tau_{1}}-\omega_{0}^{4 / 3} \gamma_{1}+\kappa \alpha_{0} \delta_{0}-\frac{3}{2} \omega_{0}^{8 / 3} \sin ^{2} i \sin 2 \theta_{0} \\
\omega_{0} \frac{\partial \gamma_{1}}{\partial \tau_{2}}= & -\frac{\partial \gamma_{0}}{\partial \tau_{1}}+2 \omega_{0}^{2 / 3} V_{1}+\omega_{0}^{5 / 3} r_{1}+\kappa \omega_{0}^{-1 / 3} \delta_{0} \beta_{0}- \\
& -\frac{3}{2} \omega_{0}^{7 / 3}\left(1+\sin ^{2} i\right)+\frac{3}{2} \omega_{0}^{7 / 3} \sin ^{2} i \cos 2 \theta_{0}+ \\
& +\frac{15}{2} \omega_{0}^{7 / 3} \sin ^{2} i \sin ^{2} \theta_{0}+2 \frac{\mathrm{d} \Omega_{0}}{\mathrm{~d} \tau_{1}} \cos i \\
\omega_{0} \frac{\partial w_{1}}{\partial \tau_{2}}= & -\frac{\partial w_{0}}{\partial \tau_{1}}-\omega_{0}^{2} z_{1}+\kappa v_{0}+\frac{3}{2} \omega_{0}^{8 / 3} \sin 2 i \sin \theta_{0}- \\
& -2 \omega_{0}^{1 / 3} \frac{\mathrm{d} \Omega_{0}}{\mathrm{~d} \tau_{1}} \sin i \sin \theta_{0} .
\end{aligned}
$$

Before presenting the multiplier equations, we will make a preliminary analysis of the state equations which leads to considerable simplification.

Equations (2.42), (2.38), and (2.41) may be combined into a single second order equation $\gamma_{1}$

$$
\begin{aligned}
\omega_{0}^{2}\left(\frac{\partial^{2} \gamma_{1}}{\partial \tau_{2}^{2}}+\gamma_{1}\right)= & \frac{3}{2} \omega_{0}^{10 / 3} \sin ^{2} i \sin 2 \theta_{0}+2 \omega_{0}^{2 / 3} \kappa \alpha_{0} \delta_{0}+ \\
& +\kappa \omega_{0}^{2 / 3}\left(\delta_{0} \frac{\partial \beta_{0}}{\partial \tau_{2}}+\beta_{0} \frac{\partial \delta_{0}}{\partial \tau_{2}}\right) .
\end{aligned}
$$

Fourier series expansions of the control terms containing $\alpha_{0}, \beta_{0}$, and $\delta_{0}$ on the right side of (2.44) contain a first harmonic which leads to unbounded $\tau_{2}$ terms in the $\gamma_{1}$ solution. However, an analysis of the expansion coefficients shows that by setting

$$
A_{31}\left(\tau_{1}\right)=B_{31}\left(\tau_{1}\right)=A_{51}\left(\tau_{1}\right)=B_{51}\left(\tau_{1}\right) \equiv 0
$$

the unbounded $\tau_{2}$ terms will disappear from the $\gamma_{1}$ solution and boundary conditions (2.36) will be satisfied.

In view of (2.37) and (2.45), the zero order multipliers become

$$
\begin{aligned}
& \lambda_{10}\left(\tau_{1}, \tau_{2}\right)=C_{11}\left(\tau_{1}\right) \omega_{0}\left(\tau_{1}\right), \\
& \lambda_{20}\left(\tau_{1}, \tau_{2}\right)=0 \\
& \lambda_{30}\left(\tau_{1}, \tau_{2}\right)=0 \\
& \lambda_{40}\left(\tau_{1}, \tau_{2}\right)=C_{11}\left(\tau_{1}\right), \\
& \lambda_{50}\left(\tau_{1}, \tau_{2}\right)=0 \\
& \lambda_{60}\left(\tau_{1}, \tau_{2}\right)=0
\end{aligned}
$$

Use of both the zero order state and multiplier solutions reduces the first order multiplier equations to the form 


$$
\begin{aligned}
\omega_{0} \frac{\partial \lambda_{11}}{\partial \tau_{2}}= & -\frac{\partial \lambda_{10}}{\partial \tau_{1}}+\omega_{0}^{5 / 3} \lambda_{21}-\omega_{0}^{5 / 3} \lambda_{51}-2 \omega_{0}^{2} C_{11} \gamma_{1}- \\
& -6 \omega_{0}^{10 / 3} C_{11} \sin ^{2} i \sin 2 \theta_{0} \\
\omega_{0} \frac{\partial \lambda_{21}}{\partial \tau_{2}}= & -\frac{\partial \lambda_{20}}{\partial \tau_{1}}+3 \omega_{0}^{8 / 3} C_{11} \sin ^{2} i \cos 2 \theta_{0} \\
\omega_{0} \frac{\partial \lambda_{31}}{\partial \tau_{2}}= & -\frac{\partial \lambda_{30}}{\partial \tau_{1}}+\omega_{0}^{2} \lambda_{61}+\frac{3}{2} \omega_{0}^{10 / 3} C_{11} \sin 2 i \cos \theta_{0} \\
\omega_{0} \frac{\partial \lambda_{41}}{\partial \tau_{2}}= & -\frac{\partial \lambda_{40}}{\partial \tau_{1}}-\omega_{0}^{2 / 3} \lambda_{21}-2 \omega_{0}^{2 / 3} \lambda_{51}-\omega_{0} C_{11} \gamma_{1} \\
\omega_{0} \frac{\partial \lambda_{51}}{\partial \tau_{2}}= & -\frac{\partial \lambda_{50}}{\partial \tau_{1}}-\omega_{0}^{1 / 3} \lambda_{11}+\omega_{0}^{4 / 3} \lambda_{41}-\omega_{0} C_{11} V_{1}-2 \omega_{0}^{2} C_{11} r_{1}+ \\
& +\frac{3}{2} \omega_{0}^{8 / 3} C_{11}\left(1-\frac{3}{2} \sin ^{2} i\right)+\frac{9}{4} \omega_{0}^{8 / 3} \sin ^{2} i \cos 2 \theta_{0} \\
\omega_{0} \frac{\partial \lambda_{61}}{\partial \tau_{2}}= & -\frac{\partial \lambda_{60}}{\partial \tau_{1}}-\lambda_{31}+\frac{3}{2} \omega_{0}^{7 / 3} C_{11} \sin 2 i \sin \theta_{0}
\end{aligned}
$$

The first order boundary conditions are

$$
\begin{aligned}
& r_{1}(0,0)=0, \quad V_{1}(0,0)=-\left.\frac{\mathrm{d} \Omega_{0}}{\mathrm{~d} \tau_{1}}\right|_{0} \cos i, \\
& \theta_{1}(0,0)=0, \quad \gamma_{1}(0,0)=0, \\
& z_{1}(0,0)=0, \quad w_{1}(0,0)=\left.\frac{\mathrm{d} \Omega_{0}}{\mathrm{~d} \tau_{1}}\right|_{0} \sin i \\
& \left\{\lambda_{11}-\omega_{0} \lambda_{41}+\omega_{0}^{2 / 3} C_{11}\left(V_{1}+\omega_{0}^{-2 / 3} \frac{\mathrm{d} \Omega_{0}}{\mathrm{~d} \tau_{1}} \cos i\right)-\right. \\
& \quad-\omega_{0}^{-1 / 3} C_{11}\left[-2 \omega_{0}^{2} r_{1}+\omega_{0}^{1 / 3} \frac{\mathrm{d} \Omega_{0}}{\mathrm{~d} \tau_{1}}+\right. \\
& \left.\left.\quad+3 \omega_{0}^{8 / 3}\left(\frac{1}{2}-\frac{3}{2} \sin ^{2} i \sin ^{2} \theta_{0}\right)\right]\right\}_{\tau_{f}}=0, \\
& \left\{\lambda_{21}-\frac{3}{2} C_{11} \omega_{0}^{5 / 3} \sin ^{2} i \sin 2 \theta_{0}\right\}_{\tau_{f}}=0, \\
& \left\{\lambda_{31}-C_{11} \omega_{0}^{-1 / 3}\left[\omega_{0}^{2} z_{1}-\omega_{0}^{1 / 3} \frac{\mathrm{d} \Omega_{0}}{\mathrm{~d} \tau_{1}} \sin i \sin \theta_{0}+\right.\right. \\
& \left.\left.\quad+\frac{3}{2} \omega_{0}^{8 / 3} \sin 2 i \sin \theta_{0}\right]\right\}_{\tau_{f}}=0, \\
& \left\{\lambda_{51}\right\}_{\tau_{f}}=0, \\
& \left\{\lambda_{61}-C_{11} \omega_{0}^{-1 / 3}\left[w_{1}-\omega_{0}^{-2 / 3} \frac{\mathrm{d} \Omega_{0}}{\mathrm{~d} \tau_{i}} \sin i \cos \theta_{0}\right]\right\}_{\tau_{f}}=0, \\
& \left\{\lambda_{41}+\frac{C_{11}}{\kappa} \omega_{0}^{-2 / 3} \sin i \frac{\mathrm{d}^{2} \Omega_{0}}{\mathrm{~d} \tau_{1}^{2}}\right\}_{\tau_{f}}=0 .
\end{aligned}
$$


The solution to the first order equations is

$$
\begin{aligned}
r_{1}\left(\tau_{1}, \tau_{2}\right)= & C_{1}\left(\tau_{1}\right)+\omega_{0}^{-2 / 3}\left[B_{1}\left(\tau_{1}\right) \sin \theta_{0}-A_{1}\left(\tau_{1}\right) \cos \theta_{0}\right]+ \\
& +\frac{1}{4} \omega_{0}^{2 / 3} \sin ^{2} i \cos 2 \theta_{0}, \\
\theta_{1}\left(\tau_{1}, \tau_{2}\right)= & \Theta_{1}\left(\tau_{1}\right)+2\left[A_{1}\left(\tau_{1}\right) \sin \theta_{0}+B_{1}\left(\tau_{1}\right) \cos \theta_{0}\right]+ \\
& +\frac{1}{8} \omega_{0}^{4 / 3} \sin ^{2} i \sin 2 \theta_{0}, \\
z_{1}\left(\tau_{1}, \tau_{2}\right)= & D_{4}\left(\tau_{1}\right) \sin i \sin \theta_{0} \\
V_{1}\left(\tau_{1}, \tau_{2}\right)= & -\frac{1}{2} \omega_{0} C_{1}\left(\tau_{1}\right)+\frac{3}{8} \omega_{0}^{5 / 3}\left(6-7 \sin ^{2} i\right)+ \\
& +\omega_{0}^{1 / 3}\left[A_{1}\left(\tau_{1}\right) \cos \theta_{0}-B_{1}\left(\tau_{1}\right) \sin \theta_{0}\right]+ \\
& +\frac{1}{2} \omega_{0}^{5 / 3} \sin ^{2} i \cos 2 \theta_{0}, \\
\gamma_{1}\left(\tau_{1}, \tau_{2}\right)= & 2 \omega_{0}^{-4 / 3} \kappa+\left[A_{1}\left(\tau_{1}\right) \sin \theta_{0}+B_{1}\left(\tau_{1}\right) \cos \theta_{0}\right]- \\
& -\frac{1}{2} \omega_{0}^{4 / 3} \sin ^{2} i \sin 2 \theta_{0}, \\
& -\frac{3}{2} \omega_{0}^{4 / 3} C_{11}\left(\tau_{1}\right) \sin 2 i \cos \theta_{0} \\
w_{1}\left(\tau_{1}, \tau_{2}\right)= & \omega_{0} D_{4}\left(\tau_{1}\right) \sin i \cos \theta_{0}, \\
\lambda_{11}\left(\tau_{1}, \tau_{2}\right)= & D_{2}\left(\tau_{1}\right)-\frac{3}{2} \omega_{0}^{5 / 3} C_{11}\left(\tau_{1}\right)\left[C_{1}\left(\tau_{1}\right)+\frac{1}{2} \omega_{0}^{2 / 3}\left(1-\frac{1}{2} \sin ^{2} i\right)\right]+ \\
& +2 \omega_{0} C_{11}\left(\tau_{1}\right)\left[A_{1}\left(\tau_{1}\right) \cos \theta_{0}-B_{1}\left(\tau_{1}\right) \sin \theta_{0}\right]+ \\
& +\omega_{0}^{2 / 3} D_{1}\left(\tau_{1}\right) \cos \left[\theta_{0}+\beta\left(\tau_{1}\right)\right] \\
& +\frac{1}{2} \omega_{0}^{4 / 3} C_{11}\left(\tau_{1}\right) \sin { }^{2} i \cos 2 \theta_{0}, \\
\lambda_{61}\left(\tau_{1}, \tau_{2}\right)= & C_{3}\left(\tau_{1}\right) \omega_{0}^{-1} \sin i \cos \left[\theta_{0}+\beta\left(\tau_{1}\right)\right] \\
\lambda_{51}\left(\tau_{1}, \tau_{2}\right)= & -2 A_{3}\left(\tau_{1}\right)-C_{11}\left(\tau_{1}\right) \kappa \omega_{0}^{-1}+D_{1}\left(\tau_{1}\right) \times \\
\lambda_{41}\left(\tau_{1}, \tau_{2}\right)= & D_{2}\left(\tau_{1}\right) \omega_{0}^{-1}+2 \omega_{0}^{-1 / 3} D_{1}\left(\tau_{1}\right) \cos \left[\theta_{0}+\beta\left(\tau_{1}\right)\right]+ \\
\lambda_{21}\left(\tau_{1}, \tau_{2}\right)= & A_{3}\left(\tau_{1}\right)+\frac{3}{2} \omega_{0}^{5 / 3} C_{11} \sin { }^{2} i \sin 2 \theta_{0}, \\
\lambda_{31}\left(\tau_{1}, \tau_{2}\right)= & C_{3}\left(\tau_{1}\right) \sin ^{2} i \sin \left[\theta_{0}+\sigma_{3}\left(\tau_{1}\right)\right] \\
& \left.+A_{1}\left(\tau_{1}\right) \cos \theta_{0}-B_{1}\left(\tau_{1}\right) \sin \theta_{0}\right]+ \\
& \\
&
\end{aligned}
$$

The boundary conditions impose the following requirements on the functions of the slow variable 


$$
\begin{aligned}
A_{1}(0)= & -\frac{1}{2}\left(3-2 \sin ^{2} i\right), \\
B_{1}(0)= & -2 \kappa, \\
C_{1}(0)= & -\frac{3}{2}\left(1-\frac{1}{2} \sin ^{2} i\right), \\
\Theta_{1}(0)= & 4 \kappa, \\
D_{4}(0)= & -\frac{3}{2} \cos i, \\
A_{3}\left(\tau_{1 f}\right)= & 0, \\
C_{3}\left(\tau_{1 f}\right)= & {\left[\omega_{0}^{5 / 3} D_{4}+\frac{9}{2} \omega_{0}^{7 / 3} \cos i\right]_{\tau_{1 f}} } \\
D_{1}\left(\tau_{1 f}\right)= & \omega_{0}^{-1}\left(\tau_{1 f}\right) \kappa, \\
D_{2}\left(\tau_{1 f}\right)= & {\left[\frac{21}{4} \omega_{0}^{7 / 3} \sin 2 i+\frac{1}{2} \omega_{0}^{7 / 3} \sin ^{2} i \cos 2 \theta_{0}+\right.} \\
& \left.\quad+\omega_{0} A_{1} \cos \theta_{0}-\omega_{0} B_{1} \sin \theta_{0}\right]_{\tau_{1 f},} \tau_{2 f}, \\
\beta\left(\tau_{1 f}\right)= & \frac{\pi}{2}-\tau_{2 f}-\Theta_{0}\left(\tau_{1 f}\right), \\
\sigma_{3}\left(\tau_{1 f}\right)= & 0 .
\end{aligned}
$$

For the first order solutions to assume the above forms without unbounded $\tau_{2}$ terms, the following equations must be satisfied:

$$
\begin{aligned}
& \frac{\mathrm{d} \omega_{0}}{\mathrm{~d} \tau_{1}}+3 \kappa \omega_{0}^{2 / 3}=0 \\
& \frac{\mathrm{d} \Omega_{0}}{\mathrm{~d} \tau_{1}}+\frac{3}{2} \omega_{0}^{7 / 3} \cos i=0 \\
& \frac{\mathrm{d} \Theta_{0}}{\mathrm{~d} \tau_{1}}+\frac{3}{2} \omega_{0}^{5 / 3} C_{1}-\frac{3}{8} \omega_{0}^{7 / 3}\left(6-7 \sin ^{2} i\right)=0 \\
& \frac{\mathrm{d} C_{11}}{\mathrm{~d} \tau_{1}}+3 \omega_{0}^{2 / 3} A_{3}=0
\end{aligned}
$$

Using (2.36) with (2.73) we find

$$
\omega_{0}\left(\tau_{1}\right)=\left(1-\kappa \tau_{1}\right)^{3} .
$$

It follows from (2.74) then that

$$
\Omega_{0}\left(\tau_{1}\right)=-\frac{3}{16 \kappa}\left[1-\left(1-\kappa \tau_{1}\right)^{8}\right] \cos i,
$$

where we have taken the $X$ and $x$ axes coincident at $\tau=0$, implying $\Omega_{0}(0)=0$. The final two quadratures (2.75), (2.76) cannot be carried out until $C_{1}$ and $A_{3}$ have been defined. 
In view of (2.77) we may find the independent variable $\tau_{2}$ from (2.3)

$$
\tau_{2}=\left(1-\frac{3}{2} \varepsilon \kappa \tau+\varepsilon^{2} \kappa^{2} \tau^{2}-\frac{1}{4} \varepsilon^{3} \kappa^{3} \tau^{3}\right) \tau
$$

or

$$
\tau_{2}=\left(1-\frac{3}{2} \kappa \tau_{1}+\kappa^{2} \tau_{1}^{2}-\frac{1}{4} \kappa^{3} \tau_{1}^{3}\right) \tau
$$

which is of the form $\tau_{2}=\omega\left(\tau_{1}\right) \tau$. Consequently, we may interpret trigonometric terms of the form $\sin \theta_{0}=\sin \left[\tau_{2}+\Theta_{0}\left(\tau_{1}\right)\right]$ as oscillations with slowly varying frequency and phase.

Finally substitution of the multiplier solutions into the control angle expansions yields

$$
\begin{aligned}
& \alpha_{0}=1, \quad \beta_{0}=0 \\
& \delta_{0}=1, \quad v_{0}=0 \\
& \alpha_{1}=0, \\
& \beta_{1}=-2 \omega_{0}^{-1 / 3} \frac{A_{3}}{C_{11}}-\kappa \omega_{0}^{-4 / 3}+\omega_{0}^{-1 / 3} \frac{D_{1}}{C_{11}} \sin \left(\theta_{0}+\beta\right), \\
& \delta_{1}=0, \quad v_{1}=\frac{C_{3}}{C_{11}} \omega_{0}^{-1} \sin i \cos \left(\theta_{0}+\sigma_{3}\right)-\frac{3}{2} \omega_{0}^{4 / 3} \sin 2 i \cos \theta_{0} .
\end{aligned}
$$

From these expressions it is obvious that to second order the optimal control is oscillatory with slowly varying frequency, phase, and amplitude.

\section{DEVELOPMENT OF THE NEAR OPTIMAL SOLUTION}

Due to the extreme complexity introduced by the inclusion of oblateness, the generation of the complete set of second-order equations was found to be impractical. Consequently, it is not possible to define the functions of $\tau_{1}$ appearing in the first-order solution in a manner which gives a second-order solution that is bounded in $\tau_{2}$. Because of this difficulty, the uniformly valid expansions for the optimal control, and optimal trajectory have not been obtained. However, by using the terminal boundary conditions (2.72) as a guide, a near optimal control program can be defined, and the uniformly valid expansion for the corresponding near-optimal trajectory can be generated. This is accomplished by choosing the functions of $\tau_{1}$ in the multiplier solution to be

$$
\begin{aligned}
A_{3}\left(\tau_{1}\right)= & 0 \\
C_{3}\left(\tau_{1}\right)= & \omega_{0}^{5 / 3}\left(\tau_{1}\right) D_{4}\left(\tau_{1}\right)+\frac{9}{2} \omega_{0}^{7 / 3}\left(\tau_{1}\right) \cos i \\
D_{1}\left(\tau_{1}\right)= & \kappa \omega_{0}^{-1}\left(\tau_{1}\right) \\
D_{2}\left(\tau_{1}\right)= & \frac{2}{4} \omega_{0}^{7 / 3}\left(\tau_{1}\right) \sin 2 i+\frac{1}{2} \omega_{0}^{7 / 3}\left(\tau_{1}\right) \sin ^{2} i \cos \theta_{0 f}+ \\
& +\omega_{0}\left(\tau_{1}\right) A_{1}\left(\tau_{1}\right) \cos \theta_{0 f}-\omega_{0}\left(\tau_{1}\right) B_{1}\left(\tau_{1}\right) \sin \theta_{0 f} \\
\beta\left(\tau_{1}\right)= & \frac{\pi}{2}-\theta_{0 f}, \\
\sigma_{3}\left(\tau_{1}\right)= & 0
\end{aligned}
$$


The resulting near optimal control is defined by

$$
\begin{array}{ll}
\alpha_{0}\left(\tau_{1}, \tau_{2}\right)=1, & \beta_{0}\left(\tau_{1}, \tau_{2}\right)=0 \\
\delta_{0}\left(\tau_{1}, \tau_{2}\right)=1, & v_{0}\left(\tau_{1}, \tau_{2}\right)=0, \\
\alpha_{1}\left(\tau_{1}, \tau_{2}\right)=0, & \beta_{1}\left(\tau_{1}, \tau_{2}\right)=-\kappa \omega_{0}^{-4 / 3}\left[1-\cos \left(\theta_{0}-\theta_{0 f}\right)\right], \\
\delta_{1}\left(\tau_{1}, \tau_{2}\right)=0, & v_{1}\left(\tau_{1}, \tau_{2}\right)=\left[\omega_{0}^{2 / 3} D_{4} \sin i+\frac{3}{4} \omega_{0}^{4 / 3} \sin 2 i\right] \cos \theta_{0} .
\end{array}
$$

This control has the same functional form as the optimal (2.80) and agrees with it at the final time. A near optimal defined in the above manner has previously been used for the case of a non-oblate planet (Jacobson and Powers, 1972), and has been found for that case to be a good approximation to the true optimal, possessing all of the major characteristics of the optimal. It is expected, therefore, that for the oblate case such a near optimal will also exhibit the characteristics of the true optimal.

In order to obtain the expansion for the near optimal trajectory, the second order state equations are needed. These, after the introduction of the zero order state solution, take the form

$$
\begin{aligned}
& \omega_{0} \frac{\partial r_{2}}{\partial \tau_{2}}=-\frac{\partial r_{1}}{\partial \tau_{1}}+\omega_{0}^{1 / 3} \gamma_{2}+\gamma_{1} V_{1} \\
& \omega_{0} \frac{\partial \theta_{2}}{\partial \tau_{2}}=-\frac{\partial \theta_{1}}{\partial \tau_{1}}+\omega_{0}^{2 / 3} V_{2}-\omega_{0}^{5 / 3} r_{2}+\omega_{0}^{4 / 3} r_{1} V_{1}+ \\
& +\omega_{0}^{7 / 3} r_{1}^{2}-\frac{1}{2} \omega_{0} \gamma_{1}^{2} \\
& \omega_{0} \frac{\partial z_{2}}{\partial \tau_{2}}=-\frac{\partial z_{1}}{\partial \tau_{1}}+w_{2} \\
& \omega_{0} \frac{\partial V_{2}}{\partial \tau_{2}}=-\frac{\partial V_{1}}{\partial \tau_{1}}-\omega_{0}^{4 / 3} \gamma_{2}+2 \omega_{0}^{2} r_{1} \gamma_{1}+\kappa\left(\alpha_{1} \delta_{0}+\alpha_{0} \delta_{1}\right)- \\
& -\left(6 \omega_{0}^{10 / 3} \sin ^{2} i \sin 2 \theta_{0}\right) r_{1}-\left(3 \omega_{0}^{8 / 3} \sin ^{2} i \cos 2 \theta_{0}\right) \theta_{1}+ \\
& +\left(-\frac{3}{2} \omega_{0}^{8 / 3}+\frac{9}{4} \omega_{0}^{8 / 3} \sin ^{2} i-\frac{9}{4} \omega_{0}^{8 / 3} \sin ^{2} i \cos 2 \theta_{0}\right) \gamma_{1}- \\
& \text { - }\left(\frac{3}{2} \omega_{0}^{10 / 3} \sin 2 i \cos \theta_{0}\right) z_{1}-\left(\frac{3}{2} \omega_{0}^{7 / 3} \sin 2 i \sin \theta_{0}\right) w_{1}- \\
& -\frac{9}{8} \omega_{0}^{4} \cos ^{2} i \sin ^{2} i \sin 2 \theta_{0}-\frac{21}{2} \omega_{0}^{4 / 3} \kappa \cos ^{2} i \text {, } \\
& \omega_{0} \frac{\partial \gamma_{2}}{\partial \tau_{2}}=-\frac{\partial \gamma_{1}}{\partial \tau_{1}}+2 \omega_{0}^{2 / 3} V_{2}+\omega_{0}^{5 / 3} r_{2}-2 \omega_{0}^{7 / 3} r_{1}^{2}-3 \omega_{0}^{4 / 3} r_{1} V_{1} \\
& -\omega_{0}^{1 / 3} V_{1}^{2}+\frac{3}{2} \omega_{0}^{7 / 3} z_{1}^{2}+\omega_{0}^{-1 / 3} \kappa\left(\delta_{1} \beta_{0}+\beta_{1} \delta_{0}\right)+ \\
& +\left(6 \omega_{0}^{3}-9 \omega_{0}^{3} \sin ^{2} i+9 \omega_{0}^{3} \sin ^{2} i \cos 2 \theta_{0}\right) r_{1}+ \\
& +\left(\frac{9}{2} \omega_{0}^{7 / 3} \sin ^{2} i \sin 2 \theta_{0}\right) \theta_{1}+\left(\frac{3}{2} \omega_{0}^{7 / 3} \sin ^{2} i \sin 2 \theta_{0}\right) \gamma_{1}+ \\
& +\left(6 \omega_{0}^{3} \sin 2 i \sin \theta_{0}\right) z_{1}+\left(\frac{3}{2} \omega_{0}^{2} \sin 2 i \cos \theta_{0}\right) w_{1}+ \\
& +\left(\frac{3}{2} \omega_{0}^{2}-\frac{9}{4} \omega_{0}^{2} \sin ^{2} i+\frac{9}{4} \omega_{0}^{2} \sin ^{2} i \cos 2 \theta_{0}\right) V_{1}+ \\
& +\frac{9}{8} \omega_{0}^{11 / 3} \cos ^{2} i\left[\left(1+\cos ^{2} i\right)+\sin ^{2} i \cos 2 \theta_{0}\right]+ \\
& +2 \frac{\mathrm{d} \Omega_{1}}{\mathrm{~d} \tau_{1}} \cos i
\end{aligned}
$$




$$
\begin{aligned}
\omega_{0} \frac{\partial w_{2}}{\partial \tau_{2}}= & -\frac{\partial w_{1}}{\partial \tau_{1}}-\omega_{0}^{2} z_{2}+3 \omega_{0}^{8 / 3} z_{1} r_{1}+\kappa v_{1}+ \\
& +\left(-\frac{9}{2} \omega_{0}^{10 / 3}+\frac{27}{4} \omega_{0}^{10 / 3} \sin ^{2} i-\frac{15}{4} \omega_{0}^{10 / 3} \sin ^{2} i \cos 2 \theta_{0}\right) z_{1}+ \\
& +\left(6 \omega_{0}^{10 / 3} \sin 2 i \sin \theta_{0}\right) r_{1}-\left(\frac{3}{2} \omega_{0}^{8 / 3} \sin 2 i \cos \theta_{0}\right) \gamma_{1}+ \\
& +\left(\frac{3}{2} \omega_{0}^{7 / 3} \sin 2 i \sin \theta_{0}\right) V_{1}-\left(2 \omega_{0}^{1 / 3} \sin i \sin \theta_{0}\right) \frac{\mathrm{d} \Omega_{1}}{\mathrm{~d} \tau_{1}}- \\
& -\frac{9}{8} \omega_{0}^{4} \cos ^{2} i \sin 2 i \sin \theta_{0}+\frac{21}{4} \kappa \omega_{0}^{4 / 3} \sin 2 i \cos \theta_{0}
\end{aligned}
$$

A uniformly valid first order expansion for the near optimal trajectory may be found if a second-order solution, bounded in $\tau_{2}$, can be generated. Such a solution is possible if the functions $A_{1}, B_{1}, C_{1}, D_{4}, \Omega_{1}$, are defined by the following differential equations

$$
\begin{aligned}
& \frac{\mathrm{d} A_{1}}{\mathrm{~d} \tau_{1}}+\left(\kappa \omega_{0}^{-1 / 3}\right) A_{1}-\frac{3}{2} \omega_{0}^{7 / 3}\left(2-\frac{5}{2} \sin ^{2} i\right) B_{1}=\frac{1}{2} \omega_{0}^{-5 / 3} \kappa \sin \theta_{0 f} \\
& \frac{\mathrm{d} B_{1}}{\mathrm{~d} \tau_{1}}+\left(\kappa \omega_{0}^{-1 / 3}\right) B_{1}+\frac{3}{2} \omega_{0}^{7 / 3}\left(2-\frac{5}{2} \sin ^{2} i\right) A_{1}=\frac{1}{2} \omega_{0}^{-5 / 3} \kappa \cos \theta_{0 f} \\
& \frac{\mathrm{d} C_{1}}{\mathrm{~d} \tau_{1}}-\left(3 \kappa \omega_{0}^{-1 / 3}\right) C_{1}=\frac{9}{2} \omega_{0}^{1 / 3} \kappa\left(1-\frac{3}{2} \sin ^{2} i\right) \\
& \frac{\mathrm{d} D_{4}}{\mathrm{~d} \tau_{1}}-\left(2 \kappa \omega_{0}^{-1 / 3}\right) D_{4}=3 \kappa \omega_{0}^{1 / 3} \cos i \\
& \frac{\mathrm{d} \Omega_{1}}{\mathrm{~d} \tau_{1}}=\frac{21}{4} \omega_{0}^{3} C_{1} \cos i+\frac{3}{2} \omega_{0}^{3} D_{4} \sin ^{2} i+ \\
& \quad+\frac{1}{16} \omega_{0}^{11 / 3}\left(36-57 \sin ^{2} i\right) \cos i
\end{aligned}
$$

The equation for $\Theta_{1}$ is not given since it contains unknown functions of $\tau_{1}$ from the second-order state solution and therefore cannot be solved at this point. As a result, the first-order term in the polar angle expansion is not known completely, and the polar angle solution must be approximated by only its zero order term.

The solution to (2.91)-(2.93) is straightforward

$$
\begin{aligned}
D_{4}\left(\tau_{1}\right)= & -\frac{3}{4}\left[\left(1-\kappa \tau_{1}\right)^{-2}+\left(1-\kappa \tau_{1}\right)^{2}\right] \cos i \\
C_{1}\left(\tau_{1}\right)= & -\frac{9}{10}\left(1-\frac{3}{2} \sin ^{2} i\right)\left(1-\kappa \tau_{1}\right)^{2}-\frac{3}{5}\left(1+\sin ^{2} i\right)\left(1-\kappa \tau_{1}\right)^{-3} \\
\Omega_{1}\left(\tau_{1}\right)= & -\frac{9}{10}\left(1+\sin ^{2} i\right) \cos i\left[\frac{1-\left(1-\kappa \tau_{1}\right)^{7}}{\kappa}\right]- \\
& -\frac{9}{64} \sin ^{2} i \cos i\left[\frac{1-\left(1-\kappa \tau_{1}\right)^{8}}{\kappa}\right]- \\
& -\left(\frac{33}{160}-\frac{1}{5} \sin ^{2} i\right) \cos i\left[\frac{1-\left(1-\kappa \tau_{1}\right)^{12}}{\kappa}\right]
\end{aligned}
$$


where the initial conditions for $D_{4}$ and $C_{1}$ are taken from (2.72) and it is assumed $\Omega_{1}(0)=0$. With $C_{1}$ known we may also now obtain $\Theta_{0}$ from (2.75) and (2.36)

$$
\begin{aligned}
\Theta_{0}\left(\tau_{1}\right)= & \frac{9}{10}\left(1+\sin ^{2} i\right)\left[\frac{1-\left(1-\kappa \tau_{1}\right)^{3}}{3 \kappa}\right]+ \\
& +\frac{3}{10}\left(12-\frac{31}{2} \sin ^{2} i\right)\left[\frac{1-\left(1-\kappa \tau_{1}\right)^{8}}{8 \kappa}\right] .
\end{aligned}
$$

The solution to (2.89), (2.90) is rather complicated, and the details are presented in Appendix $\mathrm{C}$; the final result is given below

$$
\begin{aligned}
A_{1}\left(\tau_{1}\right)= & \left(1-\kappa \tau_{1}\right)\left\{-\frac{1}{2}\left(3-2 \sin ^{2} i\right) \cos P\left[\left(1-\kappa \tau_{1}\right)^{8}-1\right]+\right. \\
& \left.+2 \kappa \sin P\left[\left(1-\kappa \tau_{1}\right)^{8}-1\right]\right\}- \\
& -\frac{1}{2} \kappa\left(1-\kappa \tau_{1}\right) \sin \left[P\left(1-\kappa \tau_{1}\right)^{8}-P-\theta_{0 f}\right] I_{C}- \\
& -\frac{1}{2} \kappa\left(1-\kappa \tau_{1}\right) \cos \left[P\left(1-\kappa \tau_{1}\right)^{8}-P-\theta_{0 f}\right] I_{S}, \\
B_{1}\left(\tau_{1}\right)= & \left(1-\kappa \tau_{1}\right)\left\{-\frac{1}{2}\left(3-2 \sin ^{2} i\right) \sin P\left[\left(1-\kappa \tau_{1}\right)^{8}-1\right]-\right. \\
& \left.-2 \kappa \cos P\left[\left(1-\kappa \tau_{1}\right)^{8}-1\right]\right\}+ \\
& +\frac{1}{2} \kappa\left(1-\kappa \tau_{1}\right) \cos \left[P\left(1-\kappa \tau_{1}\right)^{8}-P-\theta_{0 f}\right] I_{C}- \\
& -\frac{1}{2} \kappa\left(1-\kappa \tau_{1}\right) \sin \left[P\left(1-\kappa \tau_{1}\right)^{8}-P-\theta_{0 f}\right] I_{S},
\end{aligned}
$$

where

$$
\begin{aligned}
P= & \frac{3}{16 \kappa}\left(2-\frac{5}{2} \sin ^{2} i\right) \\
I_{C}= & \frac{1}{5}\left\{\frac{\cos P\left[1-\left(1-\kappa \tau_{1}\right)^{8}\right]}{\left(1-\kappa \tau_{1}\right)^{5}}-1\right\}- \\
& -\frac{8}{5} P \int_{1}^{1-\kappa \tau_{1}} x^{2} \sin P\left(1-x^{8}\right) \mathrm{d} x \\
I_{S}= & \frac{1}{5}\left\{\frac{\sin P\left[1-\left(1-\kappa \tau_{1}\right)^{8}\right]}{\left(1-\kappa \tau_{1}\right)^{5}}\right\}+ \\
& +\frac{8}{5} P \int_{1}^{1-\kappa \tau_{1}} x^{2} \cos P\left(1-x^{8}\right) \mathrm{d} x
\end{aligned}
$$

The integrals appearing in $I_{C}$ and $I_{S}$ cannot be obtained in closed form, but may be approximated by a rapidly convergent series given in Appendix $C$.

At this point we have the uniformly valid first order asymptotic expansion description of a near optimal energy increase trajectory of a low constant thrust acceleration vehicle. Moreover, since the near optimal is a first-order modification of the true optimal, we also have the uniformly valid zero order expansion for the optimal trajec- 
tory. The only unknown parameter is the final time $\tau_{f}$ which is defined implicitly by the terminal energy boundary condition.

\section{Discussion of the Solutions}

To zero order the expansions describe the solution to the optimal energy increase problem. The resulting trajectory is a spiral which maintains circular orbit conditions at each point, and which remains in the $x y$ plane of the rotating coordinate system. That plane, which slowly regresses about the inertial $Z$ axis, is then the osculating orbital plane to zero order. The optimal thrust direction is along the zero order velocity vector in the $x y$ plane, implying that to zero order, tangential thrust is optimal as in the non-oblate case (Jacobson and Powers, 1972).

Because of its similarity to the optimal, the trajectory generated by the nearoptimal control is of some interest. The first-order expansion solution exhibits an oscillatory character about the zero order circular spiral; all of the state variables oscillate with slowly varying amplitude, frequency, and phase. The near-optimal thrusting program is also oscillatory with the out of plane thrust following the out of plane velocity component and the in plane thrust remaining near the in plane velocity component. The effect of these programs on the trajectory appears to be a form of modulation of the trajectory oscillations similar to that which appears in the nonoblate case (Jacobson and Powers, 1972). In that case, it was shown that such a modulation was an important characteristic of the optimal control program; and here we see that the addition of oblateness does not change that basic characteristic.

\section{Concluding Remarks}

In this paper we have developed a general asymptotic expansion solution for the problem of near-optimal energy increase by a low thrust vehicle from an orbit about an oblate planet. The thrust acceleration of the vehicle was assumed constant and of the same order of magnitude as the initial oblateness gravitational acceleration. The resulting expansions are uniformly valid as long as the inverse square gravitational force remains dominant.

The solution is actually correct to zero order for the optimal, but the complexity of the equations prevented the development of the first order optimal as has been done for the non-oblate case. However, it is possible to generate the complete firstorder optimal for the special case of equatorial orbits and the thrust oblateness ratio, $\kappa=1$. This solution is presented in Appendix D.

Since the near-optimal control is closely related to the true optimal, the resultant near-optimal trajectory can be expected to have the same general characteristics as the actual optimal. The oscillatory behavior of the near-optimal control and its effect on the trajectory, therefore, give an indication as to the effect of an optimal control program on a trajectory. 


\section{Appendix}

\section{A. CONTROL ANGLE EXPANSIONS}

In the solution of the systems of equations, expansions for the control angle functions were employed. The relations between the terms of those expansions and the multipliers are given below. Recall

$$
\tan \phi=\frac{\lambda_{5}}{V \lambda_{4}}
$$

This may be expanded to form

$$
\tan \phi=\eta_{0}+\varepsilon \eta_{1}+\varepsilon^{2} \eta_{2}+\cdots
$$

where

$$
\begin{aligned}
\eta_{0}= & \frac{\lambda_{50}}{V_{0} \lambda_{40}} \\
\eta_{1}= & \frac{1}{V_{0} \lambda_{40}}\left[\lambda_{51}-\lambda_{50}\left(\frac{V_{1}}{V_{0}}+\frac{\lambda_{41}}{\lambda_{40}}\right)\right] \\
\eta_{2}= & \frac{1}{V_{0} \lambda_{40}}\left[\lambda_{52}-\lambda_{51}\left(\frac{V_{1}}{V_{0}}+\frac{\lambda_{41}}{\lambda_{40}}\right)+\right. \\
& \left.+\lambda_{50}\left(\frac{\lambda_{41} V_{1}}{\lambda_{40} V_{0}}+\frac{\lambda_{41}^{2}}{\lambda_{40}^{2}}+\frac{V_{1}^{2}}{V_{0}^{2}}-\frac{\lambda_{42}}{\lambda_{40}}-\frac{V_{2}}{V_{0}}\right)\right] .
\end{aligned}
$$

For the other control angle we have

$$
\tan \psi=\frac{\lambda_{6} V}{\sqrt{\lambda_{5}^{2}+\lambda_{4}^{2} V^{2}}}=\frac{\lambda_{6}}{\lambda_{4}} \cos \phi .
$$

Since $\cos \phi=\left[1+\tan ^{2} \phi\right]^{-1 / 2}$ we may write

$$
\cos \phi=\alpha_{0}+\varepsilon \alpha_{1}+\varepsilon^{2} \alpha_{2}+\cdots
$$

where

$$
\begin{aligned}
& \alpha_{0}=\frac{1}{\sqrt{1+\eta_{0}^{2}}}, \\
& \alpha_{1}=\frac{-\eta_{0} \eta_{1}}{\sqrt{1+\eta_{0}^{2}}}, \\
& \alpha_{2}=\frac{1}{2}\left[\frac{3 \eta_{0}^{2} \eta_{1}^{2}}{\left(1+\eta_{0}^{2}\right)^{5 / 2}}-\frac{\eta_{1}^{2}+2 \eta_{0} \eta_{2}}{\left(1+\eta_{0}^{2}\right)^{3 / 2}}\right] .
\end{aligned}
$$

It follows then that

$$
\tan \psi=\xi_{0}+\varepsilon \xi_{1}+\varepsilon^{2} \xi_{2}+\cdots,
$$

where 


$$
\begin{aligned}
\xi_{0}= & \frac{1}{\lambda_{40}} \alpha_{0} \lambda_{60}, \\
\xi_{1}= & \frac{1}{\lambda_{40}}\left[\alpha_{0} \lambda_{61}+\alpha_{1} \lambda_{60}-\alpha_{0} \lambda_{60} \frac{\lambda_{41}}{\lambda_{40}}\right], \\
\xi_{2}= & \frac{1}{\lambda_{40}}\left[\alpha_{0} \lambda_{62}+\alpha_{1} \lambda_{61}+\alpha_{2} \lambda_{60}+\alpha_{0} \lambda_{60}\left(\frac{\lambda_{41}^{2}}{\lambda_{40}^{2}}-\frac{\lambda_{42}}{\lambda_{40}}\right)+\right. \\
& \left.+\frac{\lambda_{41}}{\lambda_{40}}\left(\alpha_{0} \lambda_{61}+\alpha_{1} \lambda_{60}\right)\right] .
\end{aligned}
$$

Finally we may write

$$
\begin{aligned}
\cos \psi & =\left[1+\tan ^{2} \psi\right]^{-1 / 2}, \\
\sin \psi & =\cos \psi \tan \psi, \\
\sin \phi & =\cos \phi \tan \phi,
\end{aligned}
$$

and use the above expansions to get

$$
\begin{aligned}
\cos \psi & =\delta_{0}+\varepsilon \delta_{1}+\varepsilon^{2} \delta_{2}+\cdots, \\
\sin \psi & =v_{0}+\varepsilon v_{1}+\varepsilon^{2} \nu_{2}+\cdots, \\
\sin \phi & =\beta_{0}+\varepsilon \beta_{1}+\varepsilon^{2} \beta_{2}+\cdots,
\end{aligned}
$$

where

$$
\begin{aligned}
& \delta_{0}=\frac{1}{\sqrt{1+\xi_{0}^{2}}}, \\
& \delta_{1}=\frac{-\xi_{0} \xi_{1}}{\sqrt{1+\xi_{0}^{2}}}, \\
& \delta_{2}=\frac{1}{2}\left[\frac{3 \xi_{0}^{2} \xi_{1}^{2}}{\left(1+\xi_{0}^{2}\right)^{5 / 2}}-\frac{\xi_{1}^{2}+2 \xi_{0} \xi_{2}}{\left(1+\xi_{0}^{2}\right)^{3 / 2}}\right] \\
& v_{0}=\frac{\xi_{0}}{\sqrt{1+\xi_{0}^{2}}}=\frac{\xi_{1}}{\sqrt{1+\xi_{0}^{2}}}-\frac{\xi_{1} \xi_{0}^{2}}{\left(1+\xi_{0}^{2}\right)^{3 / 2}} \\
& v_{1}=\frac{\xi_{2}}{\sqrt{1+\xi_{0}^{2}}}-\frac{1}{2} \frac{2 \xi_{0}^{2} \xi_{2}-3 \xi_{0} \xi_{1}^{2}}{\left(1+\xi_{0}^{2}\right)^{3 / 2}}+\frac{3}{2} \frac{\xi_{0}^{3} \xi_{1}^{2}}{\left(1+\xi_{0}^{2}\right)^{5 / 2}} \\
& \nu_{2}=\frac{\eta_{0}}{\sqrt{1+\eta_{0}^{2}}}, \\
& \beta_{0}=\frac{\eta_{1}}{\sqrt{1+\eta_{0}^{2}}}-\frac{\eta_{1} \eta_{0}^{2}}{\left(1+\eta_{0}^{2}\right)^{3 / 2}} \\
& \beta_{2}=\frac{\eta_{2}}{\sqrt{1+\eta_{0}^{2}}}-\frac{1}{2} \frac{2 \eta_{0}^{2} \eta_{2}-3 \eta_{0} \eta_{1}^{2}}{\left(1+\eta_{0}^{2}\right)^{3 / 2}}+\frac{3}{2} \frac{\eta_{0}^{3} \eta_{1}^{2}}{\left(1+\eta_{0}^{2}\right)^{5 / 2}}
\end{aligned}
$$


B. PARTIAL DERIVATIVES OF THE ENERGY

The partial derivatives of the vehicle's energy which were required for the terminal boundary conditions are given below.

$$
\begin{aligned}
& \frac{\partial E}{\partial r}=V \frac{\mathrm{d} \Omega}{\mathrm{d} \tau} \cos i \cos \gamma-w \frac{\mathrm{d} \Omega}{\mathrm{d} \tau} \sin i \cos \theta-z\left(\frac{\mathrm{d} \Omega}{\mathrm{d} \tau}\right)^{2} \cos i \sin i \sin \theta+ \\
& +r\left(\frac{\mathrm{d} \Omega}{\mathrm{d} \tau}\right)^{2}\left(\cos ^{2} i+\sin ^{2} i \cos ^{2} \theta\right)+r\left(r^{2}+z^{2}\right)^{-3 / 2}+ \\
& +3 \varepsilon\left(r^{2}+z^{2}\right)^{-5 / 2}\left[\frac{1}{2} r+r \sin ^{2} i \sin ^{2} \theta+z \cos i \sin i \sin \theta\right]- \\
& -\frac{15}{2} \varepsilon r\left(r^{2}+z^{2}\right)^{-7 / 2}(r \sin i \sin \theta+z \cos i)^{2}, \\
& \frac{\partial E}{\partial \theta}=-V z\left(\frac{\mathrm{d} \Omega}{\mathrm{d} \tau}\right) \sin i \cos (\theta-\gamma)-r z\left(\frac{\mathrm{d} \Omega}{\mathrm{d} \tau}\right)^{2} \sin i \cos i \cos \theta+ \\
& +r w\left(\frac{\mathrm{d} \Omega}{\mathrm{d} \tau}\right) \sin i \sin \theta-r^{2}\left(\frac{\mathrm{d} \Omega}{\mathrm{d} \tau}\right)^{2} \sin ^{2} i \cos \theta \sin \theta+ \\
& +3 \varepsilon\left(r^{2}+z^{2}\right)^{-5 / 2}\left(r^{2} \sin ^{2} i \sin \theta \cos \theta+r z \sin i \cos i \cos \theta\right), \\
& \frac{\partial E}{\partial z}=-V\left(\frac{\mathrm{d} \Omega}{\mathrm{d} \tau}\right) \sin i \sin (\theta-\gamma)-r\left(\frac{\mathrm{d} \Omega}{\mathrm{d} \tau}\right)^{2} \sin i \cos i \sin \theta+ \\
& +z\left(\frac{\mathrm{d} \Omega}{\mathrm{d} \tau}\right)^{2} \sin ^{2} i+z\left(r^{2}+z^{2}\right)^{-3 / 2}+3 \varepsilon\left(r^{2}+z^{2}\right)^{-5 / 2} \times \\
& \times\left[\frac{1}{2} z+z \cos ^{2} i+r \sin i \cos i \cos \theta\right]- \\
& -\frac{15}{2} \varepsilon z\left(r^{2}+z^{2}\right)^{-7 / 2}(r \sin i \sin \theta+z \cos i)^{2}, \\
& \frac{\partial E}{\partial V}=V+r\left(\frac{\mathrm{d} \Omega}{\mathrm{d} \tau}\right) \cos i \cos \gamma-z\left(\frac{\mathrm{d} \Omega}{\mathrm{d} \tau}\right) \sin i \sin (\theta-\gamma), \\
& \frac{\partial E}{\partial \gamma}=-r V\left(\frac{\mathrm{d} \Omega}{\mathrm{d} \tau}\right) \cos i \sin \gamma+z V\left(\frac{\mathrm{d} \Omega}{\mathrm{d} \tau}\right) \sin i \cos (\theta-\gamma), \\
& \frac{\partial E}{\partial w}=w-r\left(\frac{\mathrm{d} \Omega}{\mathrm{d} \tau}\right) \sin i \cos \theta \\
& \frac{\partial E}{\partial \tau}=\left[V z \sin \gamma \sin i \cos \theta+\left(\frac{\mathrm{d} \Omega}{\mathrm{d} \tau}\right) z^{2} \sin ^{2} i \cos ^{2} \theta+V r \cos \gamma \sin i-\right. \\
& -V z \sin i \sin \theta \cos \gamma+\left(\frac{\mathrm{d} \Omega}{\mathrm{d} \tau}\right)(r \cos i-z \sin i \sin \theta)^{2}- \\
& \left.-w r \sin i \cos \theta+r^{2} \sin ^{2} i \cos ^{2} \theta\right]\left(\frac{\mathrm{d}^{2} \Omega}{\mathrm{d} \tau^{2}}\right) \text {. }
\end{aligned}
$$


C. SOLUTION OF THE EQUATIONS FOR $A_{1}\left(\tau_{1}\right), B_{1}\left(\tau_{1}\right)$

The equations form a system of linear first-order differential equations with variable coefficients.

$$
\begin{aligned}
{\left[\begin{array}{l}
\frac{\mathrm{d} A_{1}}{\mathrm{~d} \tau_{1}} \\
\frac{\mathrm{d} B_{1}}{\mathrm{~d} \tau_{1}}
\end{array}\right]=} & {\left[\begin{array}{l:l}
-\kappa \omega_{0}^{-1 / 3}\left(\tau_{1}\right) & \frac{3}{2} \omega_{0}^{7 / 3}\left(\tau_{1}\right)\left(2-\frac{5}{2} \sin ^{2} i\right) \\
-\frac{3}{2} \omega_{0}^{7 / 3}\left(\tau_{1}\right)\left(2-\frac{5}{2} \sin ^{2} i\right) & -\kappa \omega_{0}^{-1 / 3}\left(\tau_{1}\right)
\end{array}\right] \times } \\
& \times\left[\begin{array}{l}
A_{1} \\
B_{1}
\end{array}\right]+\frac{1}{2} \kappa \omega_{0}^{-5 / 3}\left(\tau_{1}\right)\left[\begin{array}{l}
\sin \theta_{0 f} \\
\cos \theta_{0 f}
\end{array}\right],
\end{aligned}
$$

which has a solution given by

$$
\left[\begin{array}{c}
A_{1}\left(\tau_{1}\right) \\
B_{1}\left(\tau_{1}\right)
\end{array}\right]=\Phi\left(\tau_{1}, 0\right)\left\{\left[\begin{array}{l}
A_{1}(0) \\
B_{1}(0)
\end{array}\right]+\int_{0}^{\tau_{1}} \Phi(0, s)\left[\begin{array}{l}
F_{1}(s) \\
F_{2}(s)
\end{array}\right] \mathrm{d} s\right\},
$$

where $\Phi\left(\tau, \tau_{0}\right)$ is the fundamental matrix of the system and

$$
\left[\begin{array}{l}
F_{1}(s) \\
F_{2}(s)
\end{array}\right]=\frac{1}{2} \kappa(1-\kappa s)^{-5}\left[\begin{array}{l}
\sin \theta_{0 f} \\
\cos \theta_{0 f}
\end{array}\right] .
$$

By straightforward means it may be shown that

$$
\begin{aligned}
& \Phi\left(\tau, \tau_{0}\right)=\left(\frac{1-\kappa \tau}{1-\kappa \tau_{0}}\right) \times \\
& \times\left[\begin{array}{l}
\cos P\left[(1-\kappa \tau)^{8}-\left(1-\kappa \tau_{0}\right)^{8}\right]-\sin P\left[(1-\kappa \tau)^{8}-\left(1-\kappa \tau_{0}\right)^{8}\right] \\
\sin P\left[(1-\kappa \tau)^{8}-\left(1-\kappa \tau_{0}\right)^{8}\right] \cos P\left[(1-\kappa \tau)^{8}-\left(1-\kappa \tau_{0}\right)^{8}\right]
\end{array}\right],
\end{aligned}
$$

where

$$
P=\frac{3}{16 \kappa}\left(2-\frac{5}{2} \sin ^{2} i\right) .
$$

Using this fundamental matrix and the initial conditions given in (2.72) we may form the solutions given in (2.98) and (2.99). The integrals $I_{C}$ and $I_{S}$ appearing in those solutions are

$$
\begin{aligned}
& I_{C}=\int_{0}^{\tau_{1}}(1-\kappa s)^{-6} \cos P\left[1-(1-\kappa s)^{8}\right] \mathrm{d} s, \\
& I_{S}=\int_{0}^{\tau_{1}}(1-\kappa s)^{-6} \sin P\left[1-(1-\kappa s)^{8}\right] \mathrm{d} s,
\end{aligned}
$$


which may be integrated by parts to obtain form (2.100). We have remaining the integrals

$$
\begin{aligned}
& \int_{1}^{1-\kappa \tau_{1}} x^{2} \sin P\left(1-x^{8}\right) \mathrm{d} x=\sin P \int_{1}^{1-\kappa \tau_{1}} x^{2} \cos P x^{8} \mathrm{~d} x- \\
& -\cos P \int_{1}^{1-\kappa \tau_{1}} x^{2} \sin P x^{8} \mathrm{~d} x \\
& \int_{1}^{1-\kappa \tau_{1}} x^{2} \cos P\left(1-x^{8}\right) \mathrm{d} x=\cos P \int_{1}^{1-\kappa \tau_{1}} x^{2} \cos P x^{8} \mathrm{~d} x+ \\
& +\sin P \int_{1}^{1-\kappa \tau_{1}} x^{2} \sin P x^{8} \mathrm{~d} x
\end{aligned}
$$

Using a change of variable we may write

$$
\begin{aligned}
& \int_{1}^{1-\kappa \tau_{1}} x^{2} \cos P x^{8} \mathrm{~d} x=\frac{1}{8} P^{-3 / 8}\left[\int_{\boldsymbol{P}}^{\infty} Z^{-5 / 8} \cos Z \mathrm{~d} Z-\right. \\
& \left.-\int_{P\left(1-\kappa \tau_{1}\right)^{8}}^{\infty} Z^{-5 / 8} \cos Z \mathrm{~d} Z\right] \text {, } \\
& \int_{1}^{1-\kappa \tau_{1}} x^{2} \sin P x^{8} \mathrm{~d} x=\frac{1}{8} P^{-3 / 8}\left[\int_{P}^{\infty} Z^{-5 / 8} \sin Z \mathrm{~d} Z-\right. \\
& \left.-\int_{P\left(1-\kappa \tau_{1}\right)^{8}}^{\infty} Z^{-5 / 8} \sin Z d Z\right] .
\end{aligned}
$$

The new integrals on the right-hand side are Generalized Fresnel Integrals which have the general form

$$
\begin{aligned}
\int_{y}^{\infty} Z^{a-1} \cos Z \mathrm{~d} Z= & C(y, a)=\Gamma(a) \cos \left(\frac{\pi a}{2}\right)-\sum_{m=0}^{\infty} \frac{(-1)^{m} y^{2 m+a}}{(2 m) !(2 m+a)} \\
\int_{y}^{\infty} Z^{a-1} \sin Z \mathrm{~d} Z= & S(y, a)=\Gamma(a) \sin \left(\frac{\pi a}{2}\right)- \\
& -\sum_{m=0}^{\infty} \frac{(-1)^{m} y^{2 m+1+a}}{(2 m+1) !(2 m+1+a)} .
\end{aligned}
$$


For our problem it can be shown that $|P|<1$ since we are only interested in $\kappa \approx 1$, and that $0<\left(1-\kappa \tau_{1}\right) \leqslant 1$. Therefore, we must evaluate the Generalized Fresnel Integrals $S\left(y, \frac{3}{8}\right), C\left(y, \frac{3}{8}\right)$ where $y=P$ or $P\left(1-\kappa \tau_{1}\right)^{8}$. Since $y$ will always be less than 1 , only a few terms of the series are needed for a good approximation to the integrals.

\section{OPTIMAL EQUATORIAL SOLUTION}

Consider the special case where $i=0$ and $\kappa=1$, this implies all motion is confined to the equatorial plane, and the thrust acceleration is equal to the initial oblateness gravitational acceleration. The second order state and multiplier equations reduce to

$$
\begin{aligned}
& \omega_{0} \frac{\partial r_{2}}{\partial \tau_{2}}=-\frac{\partial r_{1}}{\partial \tau_{1}}+\omega_{0}^{1 / 3} \gamma_{2}+\gamma_{1} V_{1} \\
& \omega_{0} \frac{\partial \theta_{2}}{\partial \tau_{2}}=-\frac{\partial \theta_{1}}{\partial \tau_{1}}+\omega_{0}^{2 / 3} V_{2}-\omega_{0}^{5 / 3} r_{2}+\omega_{0}^{4 / 3} r_{1} V_{1}+\omega_{0}^{7 / 3} r_{1}^{2}-\frac{1}{2} \omega_{0} \gamma_{1}^{2} \\
& \omega_{0} \frac{\partial z_{2}}{\partial \tau_{2}}=-\frac{\partial z_{1}}{\partial \tau_{1}}+\omega_{2} \\
& \omega_{0} \frac{\partial V_{2}}{\partial \tau_{2}}=-\frac{\partial V_{1}}{\partial \tau_{1}}-\omega_{0}^{4 / 3} \gamma_{2}+2 \omega_{0}^{2} r_{1} \gamma_{1}-\frac{3}{2} \omega_{0}^{8 / 3} \gamma_{1}-\frac{21}{2} \omega_{0}^{4 / 3} \\
& \omega_{0} \frac{\partial \gamma_{2}}{\partial \tau_{2}}=-\frac{\partial \gamma_{1}}{\partial \tau_{1}}+2 \omega_{0}^{2 / 3} V_{2}+\omega_{0}^{5 / 3} r_{2}-2 \omega_{0}^{7 / 3} r_{1}^{2}-3 \omega_{0}^{4 / 3} r_{1} V_{1}- \\
&-\omega_{0}^{1 / 3} V_{1}^{2}+\frac{3}{2} \omega_{0}^{7 / 3} z_{1}^{2}+\omega_{0}^{-1 / 3} \beta_{1}+6 \omega_{0}^{3} r_{1}+ \\
&+\frac{3}{2} \omega_{0}^{2} V_{1}+\frac{9}{4} \omega_{0}^{11 / 3}+2 \frac{\mathrm{d} \Omega_{1}}{\mathrm{~d} \tau_{1}} \\
&-\left(\frac{3}{2} \omega_{0}^{2}-2 \omega_{0}^{1 / 3} V_{1}-3 \omega_{0}^{4 / 3} r_{1}\right) \lambda_{51}-\omega_{0} \gamma_{2} C_{11} \\
& \omega_{0} \frac{\partial w_{2}}{\partial \tau_{2}}=-\frac{\partial w_{1}}{\partial \tau_{1}}-\omega_{0}^{2} z_{2}+3 \omega_{0}^{8 / 3} z_{1} r_{1}+v_{1}-\frac{9}{2} \omega_{0}^{10 / 3} z_{1} \\
& \omega_{0} \frac{\partial \lambda_{12}}{\partial \tau_{2}}=-\frac{\partial \lambda_{11}}{\partial \tau_{1}}+\omega_{0}^{5 / 3} \lambda_{22}-\omega_{0}^{5 / 3} \lambda_{52}-\left(2 \omega_{0}^{7 / 3} r_{1}-\omega_{0}^{4 / 3} V_{1}\right) \lambda_{21}- \\
&-2 \omega_{0}^{2} \gamma_{1} \lambda_{41}-\left(6 \omega_{0}^{3}-3 \omega_{0}^{4 / 3} V_{1}-4 \omega_{0}^{7 / 3} r_{1}\right) \lambda_{51}- \\
&-\left(2 \omega_{0}^{2} \gamma_{2}-6 \omega_{0}^{8 / 3} r_{1} \gamma_{1}+6 \omega_{0}^{10 / 3} \gamma_{1}-\frac{\mathrm{d}^{2} \Omega_{0}}{\mathrm{~d} \tau_{1}^{2}}\right) C_{11} \\
& \omega_{0} \frac{\partial \lambda_{42}}{\partial \tau_{2}}=-\frac{\partial \lambda_{41}}{\partial \tau_{1}}-\omega_{0}^{2 / 3} \lambda_{22}-2 \omega_{0}^{2 / 3} \lambda_{52}-\gamma_{1} \lambda_{11}+\omega_{0}^{4 / 3} r_{1} \lambda_{21}- \\
& \omega_{0} \frac{\partial \lambda_{22}}{\partial \tau_{2}=}=-\frac{\partial \lambda_{21}}{\partial \tau_{1}}, \\
& \frac{\partial \lambda_{32}}{\partial \tau_{1}}+\omega_{0}^{2} \lambda_{62}-\left(3 \omega_{0}^{8 / 3} r_{1}-\frac{9}{2} \omega_{0}^{10 / 3}\right) \lambda_{61} \\
&=
\end{aligned}
$$




$$
\begin{aligned}
\omega_{0} \frac{\partial \lambda_{52}}{\partial \tau_{2}}= & -\frac{\partial \lambda_{51}}{\partial \tau_{1}}-\omega_{0}^{1 / 3} \lambda_{12}+\omega_{0}^{4 / 3} \lambda_{42}-V_{1} \lambda_{11}+\omega_{0} \gamma_{1} \lambda_{21}- \\
& -\left(2 \omega_{0}^{2} r_{1}-\frac{3}{2} \omega_{0}^{8 / 3}\right) \lambda_{41}-\omega_{0} V_{2} C_{11}- \\
& -\left(2 \omega_{0}^{2} r_{2}-3 \omega_{0}^{8 / 3} r_{1}^{2}+6 \omega_{0}^{10 / 3} r_{1}-\omega_{0}^{-2 / 3}\left(\frac{\mathrm{d} \Omega_{0}}{\mathrm{~d} \tau_{1}}\right)^{2}\right) C_{11} \\
\omega_{0} \frac{\partial \lambda_{62}}{\partial \tau_{2}}= & -\frac{\partial \lambda_{61}}{\partial \tau_{1}}-\lambda_{32} .
\end{aligned}
$$

From (2.67), (2.72), (D.8) and the boundedness condition on the expansion terms we find

$$
\lambda_{21}\left(\tau_{1}, \tau_{2}\right)=A_{3}\left(\tau_{1}\right)=A_{3}\left(\tau_{1 f}\right)=0
$$

The boundedness conditions on $\lambda_{52}$ combined with Equations (D.7), (D.10) and (D.11) lead to the equations

$$
\begin{aligned}
& \frac{\mathrm{d}}{\mathrm{d} \tau_{1}}\left(D_{1} \cos \beta\right)-\omega_{0}^{-1 / 3}\left(D_{1} \cos \beta\right)-3 \omega_{0}^{7 / 3}\left(D_{1} \sin \beta\right)- \\
& \quad-\frac{1}{2} C_{11} A_{1}=0 \\
& \frac{\mathrm{d}}{\mathrm{d} \tau_{1}}\left(D_{1} \sin \beta\right)+3 \omega_{0}^{7 / 3}\left(D_{1} \cos \beta\right)-\omega_{0}^{-1 / 3}\left(D_{1} \sin \beta\right)- \\
& \quad-\frac{1}{2} C_{11} B_{1}=0 .
\end{aligned}
$$

Using the true optimal control program (2.80) in the state equations instead of the near optimal program (2.82) causes Equations (2.89) and (2.90) to be replaced by

$$
\begin{aligned}
& \frac{\mathrm{d} A_{1}}{\mathrm{~d} \tau_{1}}+\omega_{0}^{-1 / 3} A_{1}-3 \omega_{0}^{7 / 3} B_{1}-\frac{1}{2} \omega_{0}^{-2 / 3} \frac{D_{1}}{C_{11}} \cos \beta=0, \\
& \frac{\mathrm{d} B_{1}}{\mathrm{~d} \tau_{1}}+3 \omega_{0}^{7 / 3} A_{1}+\omega_{0}^{-1 / 3} B_{1}-\frac{1}{2} \omega_{0}^{-2 / 3} \frac{D_{1}}{C_{11}} \sin \beta=0 .
\end{aligned}
$$

With $i=0, \kappa=1$, Equation (2.91) becomes

$$
\frac{\mathrm{d} C_{1}}{\mathrm{~d} \tau_{1}}-\left(3 \omega_{0}^{-1 / 3}\right) C_{1}=\frac{9}{2} \omega_{0}^{1 / 3}
$$

From (2.76), (2.36), and (D.13) we find that

$$
C_{11}\left(\tau_{1}\right)=1
$$


The solution of (D.14)-(D.18), satisfying boundary conditions (2.72) is given below

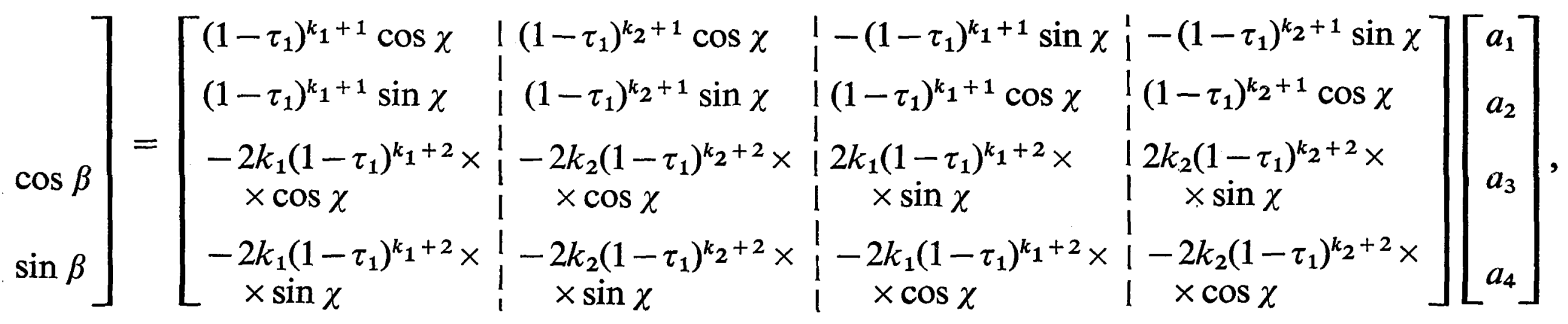

where

$$
\begin{aligned}
& \chi=\frac{3}{8}\left[\left(1-\tau_{1}\right)^{8}-1\right], \\
& k_{1}=-\frac{1}{2}[3+\sqrt{10}], \\
& k_{2}=-\frac{1}{2}[3-\sqrt{10}], \\
& a_{1}=\frac{3 k_{2}\left(1-\tau_{1 f}\right)^{k_{2}+3}+\left(1-\tau_{1 f}\right)^{-2} \sin \left[\chi\left(\tau_{1 f}\right)+\theta_{0}\left(\tau_{f}\right)\right]}{2\left(1-\tau_{1 f}\right)^{3}\left[k_{1}\left(1-\tau_{1 f}\right)^{k_{1}}-k_{2}\left(1-\tau_{1 f}\right)^{k_{2}}\right]}, \\
& a_{2}=\frac{3 k_{1}\left(1-\tau_{1 f}\right)^{k_{1}+3}+\left(1-\tau_{1 f}\right)^{-2} \sin \left[\chi\left(\tau_{1 f}\right)+\theta_{0}\left(\tau_{f}\right)\right]}{2\left(1-\tau_{1 f}\right)^{3}\left[k_{2}\left(1-\tau_{1 f}\right)^{k_{2}}-k_{1}\left(1-\tau_{1 f}\right)^{k_{1}}\right]}, \\
& a_{3}=\frac{4 k_{2}\left(1-\tau_{1 f}\right)^{k_{2}+3}+\left(1-\tau_{1 f}\right)^{-2} \cos \left[\chi\left(\tau_{1 f}\right)+\theta_{0}\left(\tau_{f}\right)\right]}{2\left(1-\tau_{1 f}\right)^{3}\left[k_{1}\left(1-\tau_{1 f}\right)^{k_{1}}-k_{2}\left(1-\tau_{1 f}\right)^{k_{2}}\right]}, \\
& a_{4}=\frac{4 k_{1}\left(1-\tau_{1 f}\right)^{k_{1}+3}+\left(1-\tau_{1 f}\right)^{-2} \cos \left[\chi\left(\tau_{1 f}\right)+\theta_{0}\left(\tau_{f}\right)\right]}{2\left(1-\tau_{1 f}\right)^{3}\left[k_{2}\left(1-\tau_{1 f}\right)^{k_{2}}-k_{1}\left(1-\tau_{1 f}\right)^{k_{1}}\right]}, \\
& \theta_{0}\left(\tau_{f}\right)=\tau_{2 f}+\frac{3}{10}\left[1-\left(1-\tau_{1 f}\right)^{3}\right]+\frac{9}{20}\left[1-\left(1-\tau_{1 f}\right)^{8}\right], \\
& C_{1}\left(\tau_{1}\right)=-\frac{9}{10}\left(1-\tau_{1}\right)^{2}-\frac{3}{5}\left(1-\tau_{1}\right)^{-3} .
\end{aligned}
$$

We now have defined all of the necessary functions for a uniformly valid first-order representation of $r, v, \gamma$, and the control angle for an optimal energy increase trajectory in the equatorial plane of an oblate planet. As in the general case, the polar angle first-order representation cannot be formed since it depends on unknown functions introduced by the second-order state solution. Comparison of the optimal and nearoptimal control programs brings out no major differences. The optimal has a slowly varying phase shift which the near optimal does not possess, and the amplitude variation of the optimal differs slightly from the near optimal.

\section{References}

Benny, D. J.: 1958, Jet Propulsion 28, 167.

Breakwell, J. V. and Rauch, H. E.: 1966, AIAA J. 4, 693.

Cole, J. D.: 1968, Perturbation Methods in Applied Mechanics, Blaisdell Publishing Company, Waltham,

Massachusetts.

Dobrowolski, A.: 1958, Jet Propulsion 28, 687. 
Jacobson, R. A. and Powers, W. F.: 1971, University of Michigan Department of Aerospace Engineering Report No. 02677-4-T, Ann Arbor, Michigan.

Jacobson, R. A. and Powers, W. F.: 1972, AIAA J. 10, 1679.

Lawden, D. F.: 1958, Astron. Acta 4, 218.

Ting, L. and Brofman, S.: 1964, Zeitschr. f. Angew. Math. Mech. 44, 417.

Tsien, H. S.: 1953, J. Am. Rocket Soc. 23, 233.

Zee, C.: 1968, Paper AD93, 19th Congress of the International Astronautical Federation, New York.

Zee, C.: 1969, Astron. Acta 14, 289. 